\title{
Lipophagy deficiency exacerbates ectopic lipid accumulation and tubular cells injury in diabetic nephropathy
}

\author{
Yachun Han ${ }^{1,3}$, Shan Xiong ${ }^{1,3}$, Hao Zhao ${ }^{1}$, Shikun Yang $\mathbb{D}^{2}{ }^{2}$, Ming Yang ${ }^{1}$, Xuejing Zhu ${ }^{1}$, Na Jiang ${ }^{1}$, Xiaofen Xiong ${ }^{1}$, Peng Gao ${ }^{1}$, Ling Wei ${ }^{1}$,
} Ying Xiao ${ }^{1}$ and Lin Sun (iD) ${ }^{1 \times}$

(c) The Author(s) 2021

Autophagy-mediated lipotoxicity plays a critical role in the progression of diabetic nephropathy (DN), but the precise mechanism is not fully understood. Whether lipophagy, a selective type of autophagy participates in renal ectopic lipid deposition (ELD) and lipotoxicity in the kidney of DN is unknown. Here, decreased lipophagy, increased ELD and lipotoxcity were observed in tubular cells of patients with DN, which were accompanied with reduced expression of AdipoR1 and p-AMPK. Similar results were found in $\mathrm{db} / \mathrm{db}$ mice, these changes were reversed by AdipoRon, an adiponectin receptor activator that promotes autophagy. Additionally, a significantly decreased level of lipophagy was observed in HK-2 cells, a human proximal tubular cell line treated with high glucose, which was consistent with increased lipid deposition, apoptosis and fibrosis, while were partially alleviated by AdipoRon. However, these effects were abolished by pretreatment with ULK1 inhibitor SBI-0206965, autophagy inhibitor chloroquine and enhanced by AMPK activator AICAR. These data suggested by the first time that autophagy-mediated lipophagy deficiency plays a critical role in the ELD and lipid-related renal injury of DN.

Cell Death and Disease (2021)12:1031; https://doi.org/10.1038/s41419-021-04326-y

\section{INTRODUCTION}

Diabetic nephropathy (DN) is a severe complication of diabetes mellitus and has become the most common primary disease leading to end-stage renal disease worldwide [1]. The importance of tubular injury in the progression of $\mathrm{DN}$ and the role of inflammation, mitochondrial oxidative stress and autophagy disorders in the pathogenesis of DN was confirmed in our previous studies [2, 3]. Emerging evidence suggests that the dyslipidemia in patients with DN was associated with estimated glomerular filtration rate (GFR), inflammation, fibrosis and disease progression [4, 5]. High-plasma triacylglycerols(TG) and nonesterified fatty acids (NEFAs) results in the lipid accumulation and lipotoxicity in non-adipose tissues $[6,7]$. Moreover, renal cells were damaged by ectopic lipid deposition (ELD) associated with lipotoxicity via activating inflammation, reactive oxygen species (ROS) production, mitochondrial dysfunction and cell-death in kidney of DN [4, 8-12]. However, the precise mechanism through which lipid disorders impact the kidney damage in DN remains unclear.

Selective autophagy, lipophagy, plays a key role in regulating the synthesis and degradation of intracellular lipid droplets (LDs) in hepatocytes [13], macrophage foam cells [14], etc. Lipophagy refers to that when LDs are sequestered by autophagosomes, which fuse with lysosomes to form autolysosomes, TG and cholesteryl esters in LDs are then degraded by lysosomal hydrolases in autolysosomes into free fatty acids (FFAs) that are recycled back into the cytosol for mitochondrial $\beta$-oxidation $[15,16]$. The activation of lipophagy was dependent on the environment and energy requirement of different types of cells [17], such as during prolonged starvation and the early phase of lipid challenge [16, 18]. Defective lipophagy were reported to be linked with liver steatosis, obesity, atherosclerosis and the metabolic syndrome of aging [14, 18-21]. Interestingly, increased lipophagy may relieve insulin resistance by decreasing lipid accumulation in adipocytes in patients with type 2 diabetes [22]. However, the level of lipophagy in kidney tissues in DN and whether it is involved in the kidney injury is unknown.

In this study, lipophagy deficiency was observed in the tubular cells of patients with $\mathrm{DN}$ and $\mathrm{db} / \mathrm{db}$ mice, which was accompanied by significantly ELD, oxidative stress, apoptosis. These damages were ameliorated in tubular cells as the levels of lipophagy was increased by AdipoRon administration, while the effects were blocked partially by AdipoR1 siRNA, autophagy inhibitor, ULK1 inhibitor and enhanced by AMPK activator. These data demonstrated by first time that disrupted lipophagy modulated by AdipoR1/AMPK pathway plays a key role in ELD and lipotoxicity in tubular cells of DN.

\section{RESULTS}

Renal injury and ELD were associated with lipophagy deficiency in patients with DN

Elevated blood glucose, hemoglobin $\mathrm{A} 1 \mathrm{c}(\mathrm{HbA} 1 \mathrm{C}), \mathrm{TG}$, total cholesterol (TC), low-density lipoprotein cholesterol (LDL-C) and

\footnotetext{
'Department of Nephrology, The Second Xiangya Hospital, Central South University, Key Laboratory of Kidney Disease and Blood Purification, Changsha, Hunan, China.

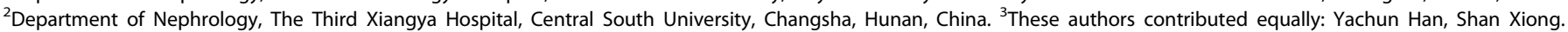
冈email: sunlin@csu.edu.cn

Edited by Professor Alessandro Finazzi-Agrò
}

Received: 20 April 2021 Revised: 14 October 2021 Accepted: 15 October 2021

Published online: 30 October 2021 
Table 1. Clinical characteristics of the patients with DN and the patients with minor lesions who did not have diabetes.

\begin{tabular}{|c|c|c|c|}
\hline & Control & DN & $p$ \\
\hline BMI $\left(\mathrm{kg} / \mathrm{m}^{2}\right)$ & $23.1 \pm 3.1$ & $24.7 \pm 4.2$ & 0.25 \\
\hline Blood glucose (mmol/L) & $4.68 \pm 0.52$ & $8.6 \pm 0.23$ & $<0.0001$ \\
\hline HBA1c (\%) & $4.75 \pm 0.46$ & $8.2 \pm 0.53$ & $<0.0001$ \\
\hline Total cholesterol (mmol/L) & $6.31 \pm 0.14$ & $7.24 \pm 0.19$ & $<0.0001$ \\
\hline Triglyceride (mmol/L) & $2.65 \pm 0.38$ & $4.22 \pm 1.6$ & 0.0009 \\
\hline $\mathrm{LDL}(\mathrm{mmol} / \mathrm{L})$ & $2.1 \pm 0.64$ & $2.9 \pm 0.88$ & 0.0082 \\
\hline ALT (IU/L) & $18.3 \pm 4.3$ & $17.8 \pm 5.4$ & 0.7811 \\
\hline AST (IU/L) & $24.1 \pm 8.7$ & $23.8 \pm 7.67$ & 0.9209 \\
\hline Albumin $(g / L)$ & $39.1 \pm 3.5$ & $32.3 \pm 1.9$ & $<0.0001$ \\
\hline BUN (mmol/L) & $5.81 \pm 0.41$ & $6.03 \pm 0.22$ & 0.07777 \\
\hline $\operatorname{Scr}(\mu \mathrm{mol} / \mathrm{L})$ & $92.4 \pm 8.9$ & $95.17 \pm 7.54$ & 0.3656 \\
\hline UA $(\mu \mathrm{mol} / \mathrm{L})$ & $346 \pm 67$ & $409 \pm 83$ & 0.0299 \\
\hline Urine protein $(g / 24 h)$ & $3.14 \pm 1.31$ & $4.38 \pm 1.09$ & 0.0088 \\
\hline $\begin{array}{l}\text { Red blood cells count in } \\
\text { urine sediment }(/ \mathrm{mL})\end{array}$ & $15010 \pm 998$ & $11003 \pm 1034$ & $<0.0001$ \\
\hline Systolic pressure $(\mathrm{mmHg})$ & $119.12 \pm 4.5$ & $148.19 \pm 2.5$ & $<0.0001$ \\
\hline Diastolic pressure $(\mathrm{mmHg})$ & $82.14 \pm 1.67$ & $96.13 \pm 2.07$ & $<0.0001$ \\
\hline
\end{tabular}

Values are the mean \pm SD.

HBA1C glycosylated hemoglobin, $L D L$ low-density lipoprotein, $A L T$ alanineaminotransferase, AST aspartate aminotransferase, BUN blood urea nitrogen, Scr serum creatinine, UA uric acid.

24-h urine protein and decreased albumin were observed in patients with DN (Table 1). Significant typical nodular glomerulosclerosis, basement membrane thickening, focal tubular atrophy, interstitial fibrosis, severe tubular injury and obvious lipid deposition occurred in the kidney of patients with DN (Fig. 1A-D). Positive correlations were observed in tubular interstitial damage and lipid deposition $(r=0.872)$, tubular interstitial damage and adipose differentiation related protein (ADRP) expression $(r=$ 0.907), lipid deposition and ADRP expression ( $r=0.807)$ in kidney tissues of patients. Transmission Electron Microscope (TEM) detection showed that some LDs were sequestrated by doublemembrane organelles, namely, autophagosomes, in both groups. More LDs and less lipophagy were represented in the kidney of DN, which indicated that lipid deposition and insufficient lipophagy may be closely related to pathological injury in the kidney of DN (Fig. 1E).

\section{The expression of AdipoR1, p-AMPK, and LC3B in the kidneys of patients with DN were noticeably decreased and the AdipoR1 expression was correlated with renal function as well as autophagosome assembly}

Moreover, considering that AdipoR1 is involved in the regulation of lipid metabolism and autophagy, AdipoR1 was detected to explore the mechanism of lipophagy deficiency in the DN kidney tissues. Immunofluorescence (IF) analysis revealed that AdiopR1 expression was reduced in kidneys of patients with DN (Fig. 1F, G). Additionally, AMPK is an important modulator of autophagy and a downstream regulator of AdipoR1, the level of its phosphorylation in the kidney and its relationship with lipid deposition and tubular injury were detected. The expression of p-AMPK and LC3B in the kidneys of patients with DN was noticeably decreased (Fig.1F, G). Here, AdipoR1 expression was negatively correlated with lipid deposition ( $r=-0.653)$, ADRP expression $(r=-0.683)$, and positively correlated with p-AMPK expression $(r=0.849)$ and LC3B expression $(r=0.804)$. P-AMPK expression was negatively correlated with tubular interstitial damage $(r=-0.868)$, lipid deposition ( $r=-0.774)$, ADRP expression $(r=-0.863)$, but positively correlated with LC3B expression $(r=0.893)$. LC3B expression was negatively correlated with tubular interstitial damage $(r=$ $-0.761)$ and lipid deposition $(r=-0.618)$. Meanwhile, Gene Ontology analysis was carried out, and the results showed that some of the genes positively coexpressed with AdipoR1 (correlation coefficient $\geq 0.60$ ) were enriched in metabolic pathway and autophagosome assembly according to Kyoto Encyclopedia of Genes and Genomes (KEGG) pathway (Fig. 2A) and biology process analysis (Fig. 2B), respectively. Additionally, real-time PCR detection determined the expression of AdipoR1, AdipoR2 and AdipoQ in the HK-2 cells, BUMPT cells (a mouse proximal tubulederived cell line) and mouse podocytes, AdipoR1 and AdipoR2 were expressed in all of these cells' line, while AdipoQ was expressed in BUMPT cells and mouse podocytes (Fig. 2C). Moreover, according to the Nephroseq database, AdipiR1 expression was positively correlated with GFR ( $r=0.708$, Fig. 2D) and negatively correlated with serum creatinine (Scr) level $(r=-0.644$, Fig. 2E) in patients with $\mathrm{DN}$, indicating that the expression of AdipoR1 was closely correlated with renal function in patients with DN.

\section{Increased lipophagy by AdipoRon ameliorates renal injury, lipid deposition, oxidative stress, and apoptosis in the kidney of $\mathrm{db} / \mathrm{db}$ mice}

Treatment of $\mathrm{db} / \mathrm{db}$ mice with AdipoRon for 4 weeks, the body weight, kidney weight and Scr level of $\mathrm{db} / \mathrm{db}$ mice showed no significant change from those of the untreated group. Blood glucose, HbA1C, TG and 24-h albuminuria were increased in $\mathrm{db} / \mathrm{db}$ mice compared with control mice, but these changes were all reversed by AdipoRon treatment. Serum adiponectin levels decreased obviously in $\mathrm{db} / \mathrm{db}$ mice compared with control mice, and there was no significant difference in adiponectin levels between the $\mathrm{db} / \mathrm{db}$ mice treated with AdipoRon and the untreated $\mathrm{db} / \mathrm{db}$ mice (Table 2).

In addition, glomerular hypertrophy, mesangial cells and matrix significantly increased in some glomeruli and some dilated or atrophied tubules and renal interstitial fibrosis were noted in the kidneys of $\mathrm{db} / \mathrm{db}$ mice, which were all partially alleviated following AdipoRon treatment (Fig. 3A, C). Additionally, cell apoptosis and oxidative stress in the kidneys were enhanced in $\mathrm{db} / \mathrm{db}$ mice compared with control mice, while were attenuated by AdipoRon (Fig. 3B, D). Moreover, AdipoRon administration decreased intrarenal lipid concentrations, which were represented by the decreased renal NEFA and TG in $\mathrm{db} / \mathrm{db}$ mice (Fig. 3E, F), while there was no significant alteration in TC (Fig. 3G). Further analysis revealed lipid deposition in the kidneys of $\mathrm{db} / \mathrm{db}$ mice via IHC for ADRP detection and Oil-Red O staining. AdipoRon dramatically ameliorated this enhancement (Fig. 3H, I). Ultrastructural analysis revealed an increased number of LDs in the kidneys of $\mathrm{db} / \mathrm{db}$ mice, and fewer LDs in the kidneys of $\mathrm{db} / \mathrm{db}$ mice treated with AdipoRon. Importantly, in the kidneys of control mice and $\mathrm{db} / \mathrm{db}$ mice treated with AdipoRon, clear autophagosomes or autolysosomes that engulfed LD were noted (Fig. $3 \mathrm{~J}, \mathrm{~K}$ ).

\section{AdipoRon upregulated the expression of p-AMPK, p-ULK1 and autophagosome-associated proteins, while reduced fibrosis in the kidney of $\mathrm{db} / \mathrm{db}$ mice}

The decreased expression of AdipoR1, p-AMPK and LC3B and increased $p$-mTOR level in $\mathrm{db} / \mathrm{db}$ mice were all partially reversed by AdipoRon treatment (Fig. 4A-D). Similar alterations were also showed in autophagosome-associated proteins such as beclin1 (BECN1), autophagy related 5 (ATG5) and LC3-II in the LD fraction. Sequestosome 1 (SQSTM1) was not detected. The purification degree of the LD fraction was 
A

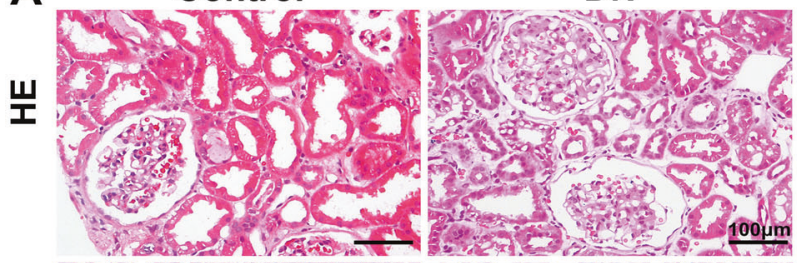

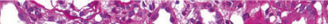
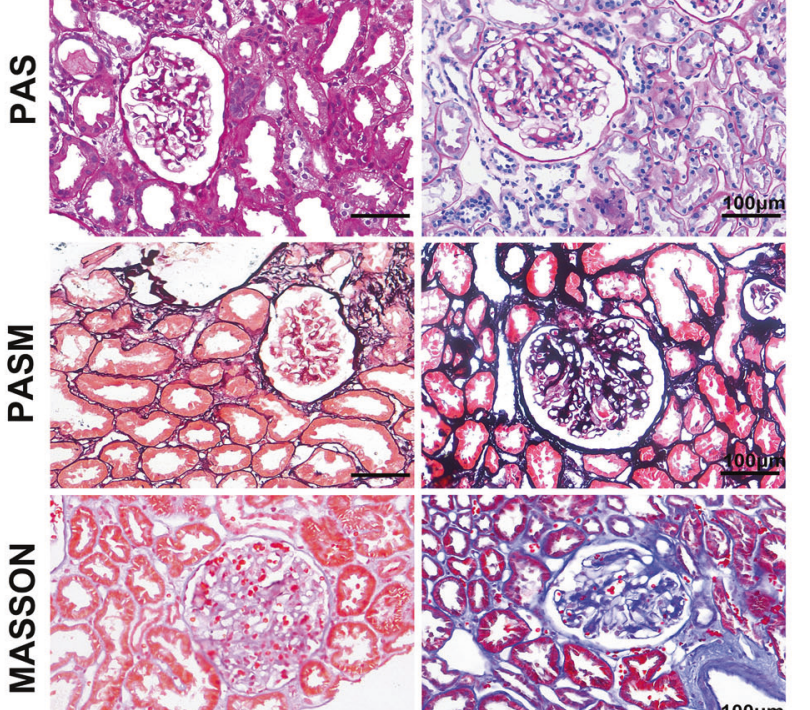

$21 \times 1=$

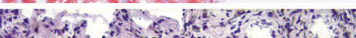
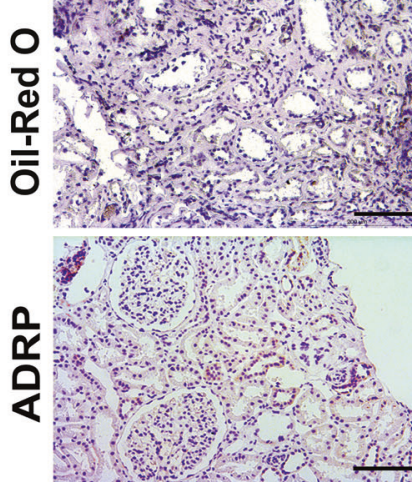

E
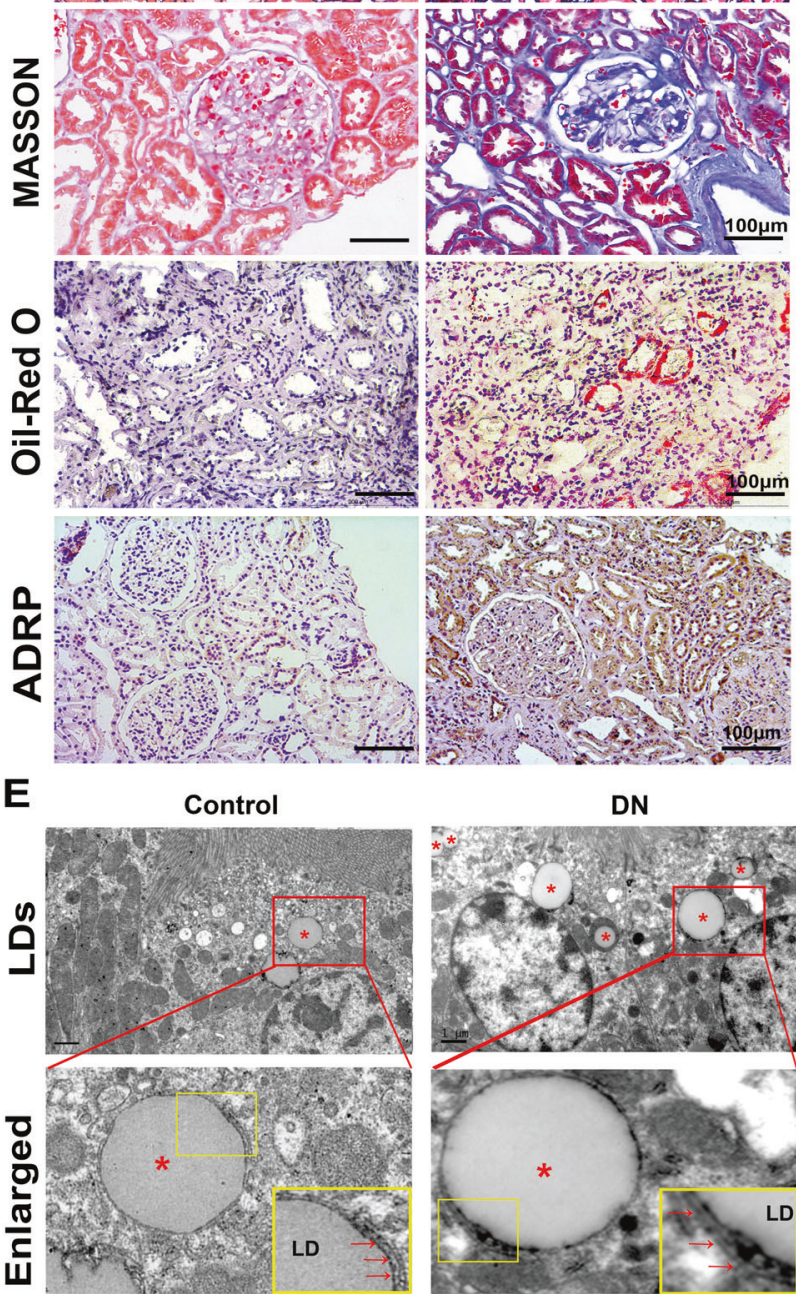

DN

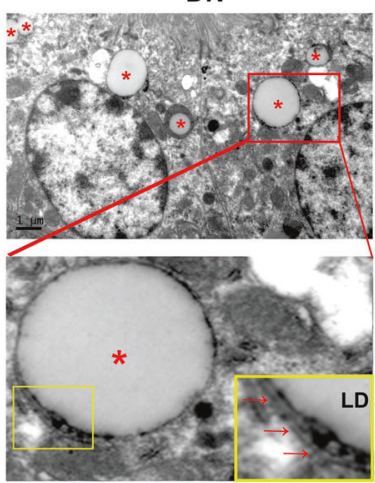

C
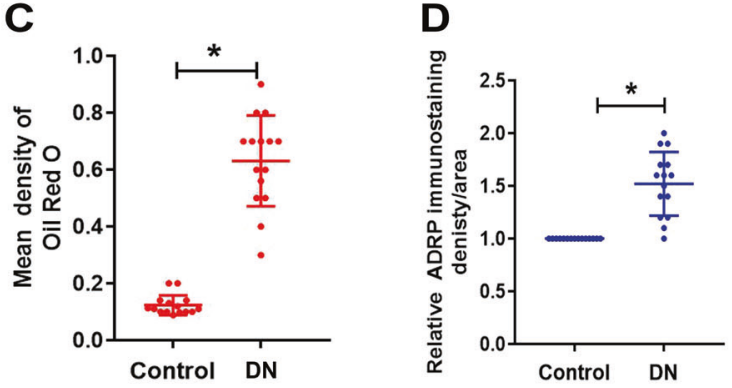

$\mathbf{F}$

B
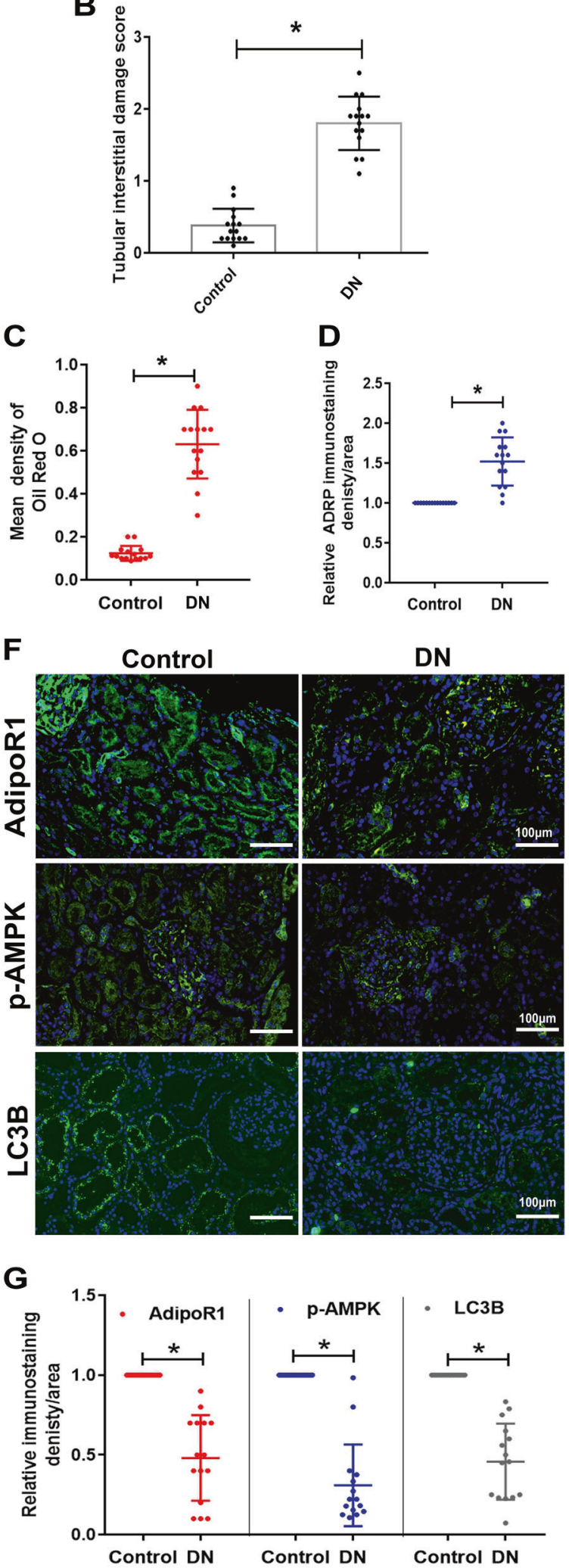

Fig. 1 Decreased lipophagy and pathological changes in the kidney tissues of patients with DN. HE, PAS, PASM, Masson trichrome, Oil-red $O$ staining and immunohistochemical analysis of ADRP in the renal biopsy tissues of patients with DN and control (magnification, $\times 200)(\mathbf{A})$. Tubular interstitial damage score (B). Quantification of Oil-Red O density (C) and ADRP expression (D). Ultrastructural analysis of lipid deposition in the kidney tissues of patients with DN and control (magnification, $\times 10,000) *$ indicates LDs, red arrows indicate doublemembrane autophagic vacuoles containing lipids (E). IF analysis and semiquantification of AdipoR1, p-AMPK and LC3B in the kidney tissues of patients with DN and control individuals $(\mathbf{F}, \mathbf{G})$. Values are the mean \pm SD; ${ }^{*} p<0.05$ versus control. $n=15$. 
A

AdiopR1 Coexpression Genes GO analysis: KEGG pathway

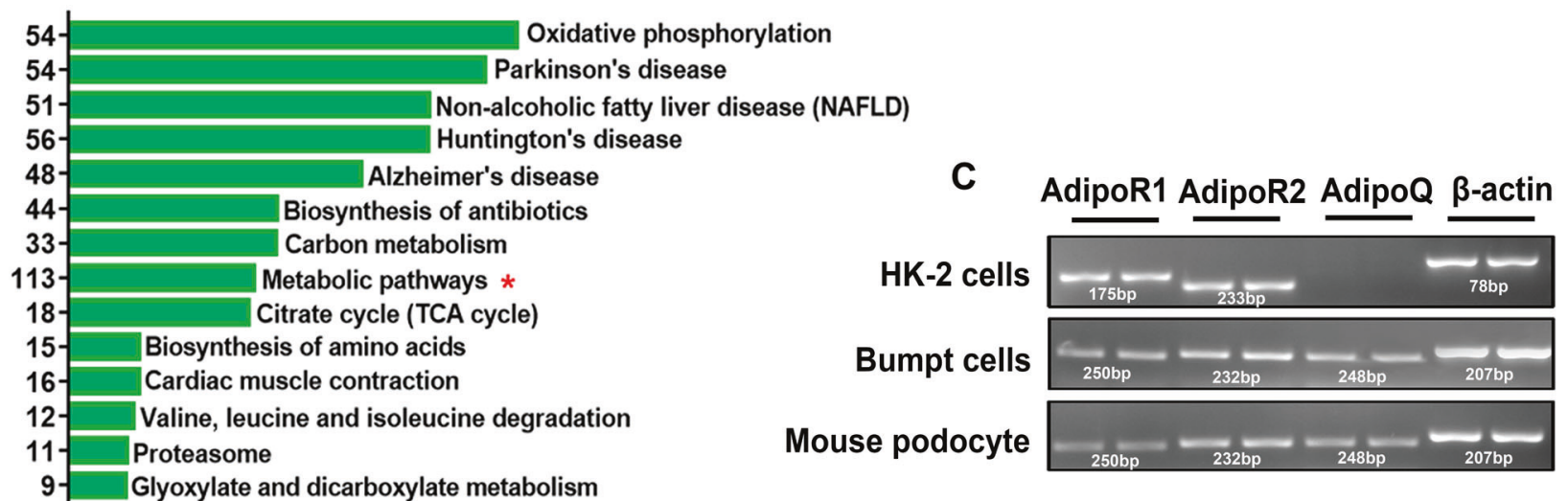

Glyoxylate and dicarboxylate metabolism

13- Glycolysis / Gluconeogenesis

2-Oxocarboxylic acid metabolism

Propanoate metabolism
Pyruvate metabolism
Synaptic vesicle cycle

Enriched score -Log10 ( $p$ value)

B

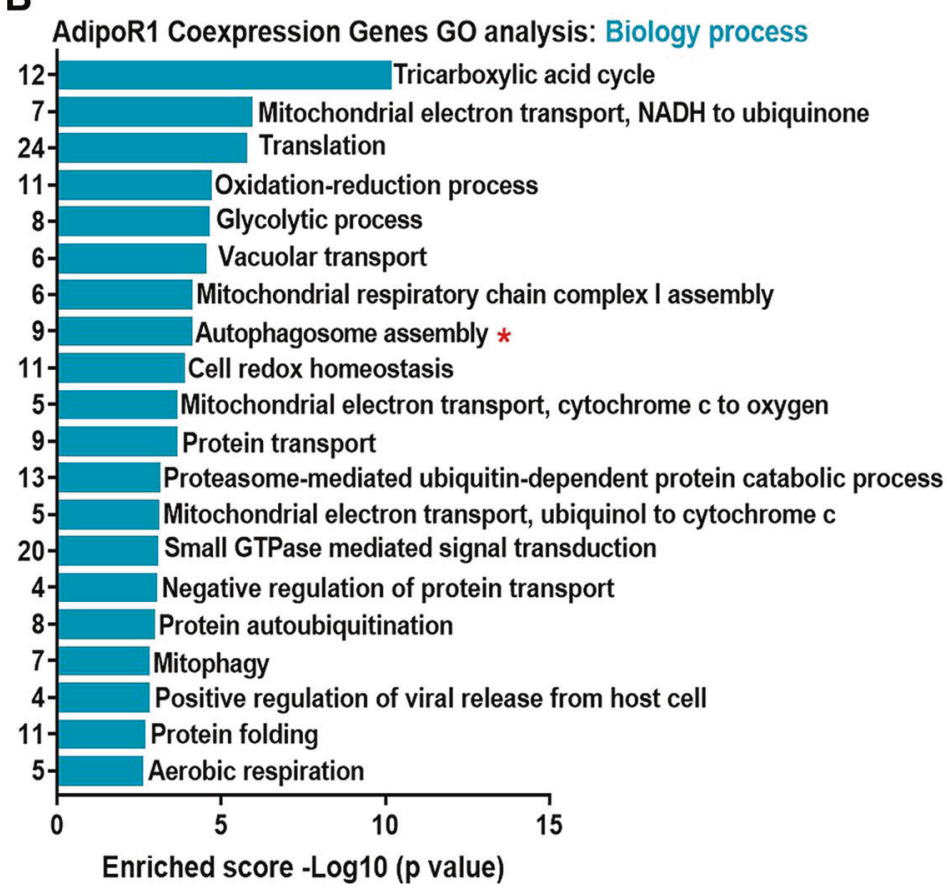

D

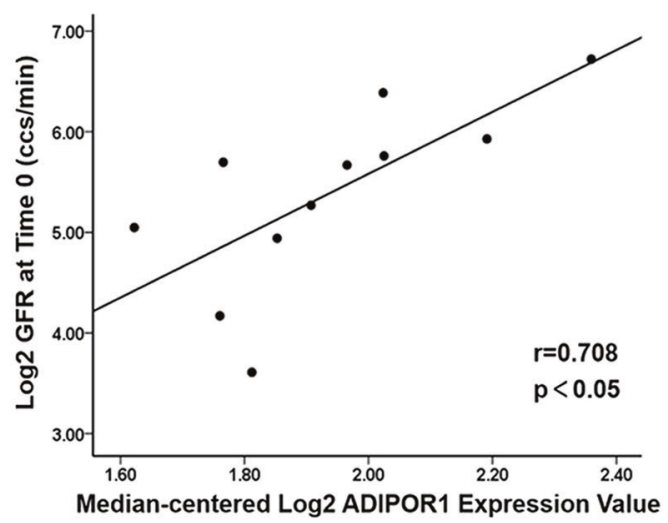

E

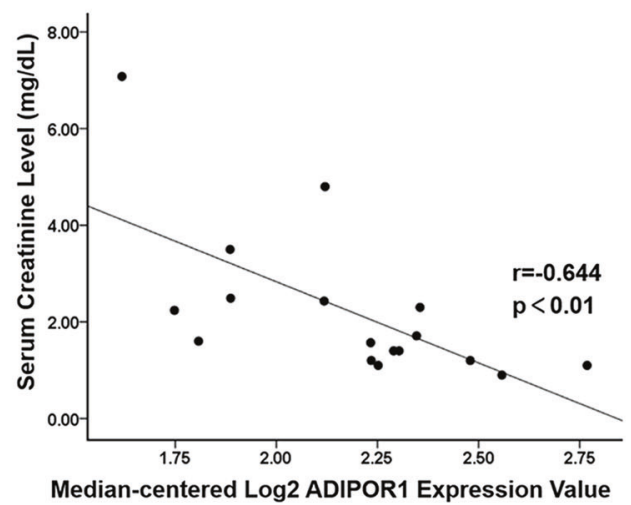

Fig. 2 Analysis of AdipoR1 genes. GO analysis of genes coexpressed with AdipoR1 (positive correlation coefficient $\geq 0.6$ ) according to KEGG pathway (A) and Biology process (B). Detection of AdipoR1, AdipoR2 and AdipoQ by PCR amplification of DNA from HK-2 cells, BUMPT cells and mouse podocytes (C). Correlation of AdipoR1 expression and GFR (D) and serum creatinine level (E) in patients with DN and control individuals from the Nephroseq database. Values are the mean \pm SD; $r$ : correlation coefficient; ${ }^{*} p<0.05$ versus control.

determined by the abundant ADRP expression and the absence of GAPDH (Fig. 4E). Fibronectin (FN)and Collagen I were reduced by AdipoRon treatment (Fig. 4F, G). Protein alterations in ADRP, sterol regulatory element binding protein1 (SREBP-1), FN and Collagen I were further confirmed by WB analysis (Fig. 4H, I).
AdipoRon reversed the lipophagy alteration and lipid deposition in HK-2 cells exposed to high-glucose (HG) ambience

The co-localization studies of autophagosomes, lysosomes and LDs were performed to identify whether LDs are associated with lipophagy. AdipoRon treatment increased autophagosomes 
Table 2. Characteristics of the different groups' mice.

\begin{tabular}{|llllr|}
\hline Group & $\mathbf{d b} / \mathbf{m}$ & $\mathbf{d b} / \mathbf{d b}$ Cont & $\mathbf{d b} / \mathbf{d b}+$ AdipoRon & $\boldsymbol{p}^{*}$ \\
\hline$n$ & 12 & 12 & 12 & $\boldsymbol{p}^{\#}$ \\
\hline Body weight $(\mathrm{g})$ & $29.8 \pm 1.5$ & $53.3 \pm 5.2$ & $51.5 \pm 3.2$ & $<0.0001$ \\
\hline Kidney weight $(\mathrm{mg})$ & $203 \pm 5.8$ & $204.7 \pm 7.3$ & $203 \pm 9.2$ & 0.4531 \\
\hline Blood glucose $(\mathrm{mmol} / \mathrm{L})$ & $8.5 \pm 0.5$ & $29.89 \pm 3.6$ & $21.7 \pm 3.7$ & 0.8471 \\
\hline HbA1c $(\%)$ & $4.1 \pm 0.2$ & $9.2 \pm 0.4$ & $8.6 \pm 0.1$ & $<0.0001$ \\
\hline Triglyceride $(\mathrm{mmol} / \mathrm{L})$ & $0.98 \pm 0.2$ & $1.96 \pm 0.2$ & $1.63 \pm 0.3$ & $<0.0001$ \\
\hline Total cholesterol $(\mathrm{mmol} / \mathrm{L})$ & $1.43 \pm 0.2$ & $3.46 \pm 0.3$ & $3.2 \pm 0.4$ & $<0.0001$ \\
\hline Scr $(\mu$ mol/L)) & $16.6 \pm 2.9$ & $19.1 \pm 3.6$ & $17.8 \pm 3.3$ & $<0.0001$ \\
\hline 24h albuminuria $(\mathrm{mg} / \mathrm{day})$ & $10.17 \pm 1.7$ & $222.8 \pm 35.2$ & $157.4 \pm 20.7$ & 0.0017 \\
\hline Serum adiponectin $(\mu \mathrm{g} / \mathrm{mL})$ & $10.9 \pm 0.8$ & $4.6 \pm 0.4$ & $4.7 \pm 0.5$ & 0.13 \\
\hline
\end{tabular}

Values are the mean \pm SD.

$p^{*}: \mathrm{db} / \mathrm{m}$ versus $\mathrm{db} / \mathrm{db} ; p^{\#}: \mathrm{db} / \mathrm{db}$ versus $\mathrm{db} / \mathrm{db}+$ AdipoRon.

HBA1c glycosylated hemoglobin, $\mathrm{Scr}$ serum creatinine.

(stained by monodansylca- daverine, MDC) and lysosomes (stained by Lyso-tracker), while decreased the number of LDs (stained by Bodipy). Moreover, AdipoRon treated cells exhibited more co-localization of LDs with autophagosomes and lysosomes (increased white stained regions). Additionally, inhibition of lysosomal degradation of LDs that sequestered autophagosomes by chloroquine (CQ) enhanced co-localization of LDs with autophagosomes (Fig. 5A). The upregulation of LC3-II levels in isolated LD fraction from HK-2 cells exposed to HG conditions with AdipoRon and CQ treatment were confirmed by WB analysis (Fig. 5B, E). Similar results were also seen in the expression of transcription factor $\mathrm{EB}$ (TFEB) in nuclear protein extracted from HK2 cells under HG condition pretreated with AdipoRon (Fig. 5C, D). Additionally, a decreased expression of AdipoR1, p-AMPK and p-ULK1 as well as an increasing level of $p$-mTOR in total cell protein from HK-2 cells exposed to HG were found, while were reversed when pretreated with AdipoRon (Fig. 6A, B).

\section{The effects of AdipoRon on increasing lipophagy, reducing ELD and fibrosis in HK-2 cells were partially blocked by AdipoR1 siRNA and ULK1 inhibitor, while enhanced by AMPK activator}

Effects of AdipoRon were abolished by pretreatment with AdipoR1 siRNA or ULK1 inhibitor SBI-0206965 and reinforced by AMPK activator AICAR (Fig. 6C, D). Similar alterations in BECN1, ATG5 and LC3-II were also observed in the LD fraction isolated from HK-2 cells exposed to $\mathrm{HG}$ after pretreated with AdipoR1 siRNA, SBI-0206965 and AICAR (Fig. 6E).

When intracellular LDs were engulfed by autophagosomes, ADRP was removed and dissociated from RAB7, the protein on the LD surface that subsequently interacts with autophagy proteins, such as SQSTM1/p62 and LC3-II [16]. The co-localization of RAB7 and LC3B, BECN1 and LDs (Bodipy stained) was decreased in HK-2 cells under HG conditions, and AdipoRon administration reversed these changes. However, these effects were partially abolished by pretreatment with AdipoR1 siRNA, SBI-0206965 and enhanced by AICAR (Fig. 7A, B). Similar alterations were also found in the intracellular lipid deposition (Fig. 7C, D), which was accompanied by the protein alterations of ADRP, SREBP-1, FN and Collagen I (Fig. 7E, F). Overall, AdipoRon activated AdipoR1/AMPK pathway to increase lipophagy that alleviated lipid deposition and fibrosis in HK-2 cells exposed to HG conditions pathway.

\section{DISCUSSION}

The present study demonstrated by first that the lipophagy deficiency in tubular cells was related to the ELD-associated kidney damage in both patients and mice with DN. Increasing the level of lipophagy by administration of AdipoRon through AdipoR1/AMPK pathway, which ameliorates lipotoxicityassociated tubular injury, oxidative stress and apoptosis. These data indicate that lipophagy dysfunction contributes to ELDinduced kidney injury in DN.

Lipophagy, a type of selective autophagy that sequesters LDs in autophagosomes for degradation by lysosomes [15]. It has been reported that lipophagy was associated with hepatic disorders, including liver steatosis, nonalcoholic fatty liver disease, and liver fibrosis $[23,24]$. The methods of identifying lipophagy include OilRed O, BODIPY $493 / 503$ or HCS LipidTOX staining, triglyceride content determination and TEM [15]. It also has been demonstrated that lipophagy is induced to breakdown LDs to maintain energy homeostasis under prolonged starvation in proximal tubule cells $[15,25]$. Furthermore, lipophagy was inhibited under nutrient abundance since FFAs were not needed as an energy source for cells [26]. Chronic lipid exposure such as ELD might be a contributor to lipophagy disorder in type 2 diabetes [22]. Moreover, previous studies by our and other group have found that renal ELD and its associated lipotoxicity in the kidney are the main causes of the development of obesity-related nephropathy and type 2 DN [4, 27-29]. Abnormal autophagy was found to participate in kidney injury in DN in vivo and in vitro $[25,30,31]$. However, whether autophagy-mediated lipophagy involved in the pathogenesis and development of chronic kidney disease including DN still needs to be discussed. Here, we demonstrated that the reduced lipophagy and lipid deposition in the renal tissues of patients with DN were positively correlated with lipotoxicity induced tubular injury (Fig. 1).

On the other hand, decreased renal AdipoR1 expression was accompanied by increased renal TG levels in diabetic rats [32], while increasing AdipoR1 expression ameliorated lipotoxicity [33]. Here, we confirmed the expression of AdipoR1 in tubular cells, and Gene Ontology analysis showed that the genes positively correlated with AdipoR1 expression were enriched in metabolic pathways and autophagy formation, and the expression level of AdipoR1 was correlated with renal function in DN patients (Fig. 2). In addition, AdipoRon, an AdipoR1 activator, which can enhance glucose and lipid metabolism and inhibits lipotoxicity of diabetic mice through AdipoR1/ AMPK pathway [10, 34], it also might promote autophagy in renal epithelial cells [35]. However, it is not clear that whether enhanced autophagy-mediated lipophagy by AdipoRon might alleviate lipotoxicity induced renal injury in DN. In this study, we found that levels of lipophagy in kidney of diabetic mouse was increased by AdipoRon, which was accompanied by decrease in proteinuria and renal lipid contents levels as well as 
A
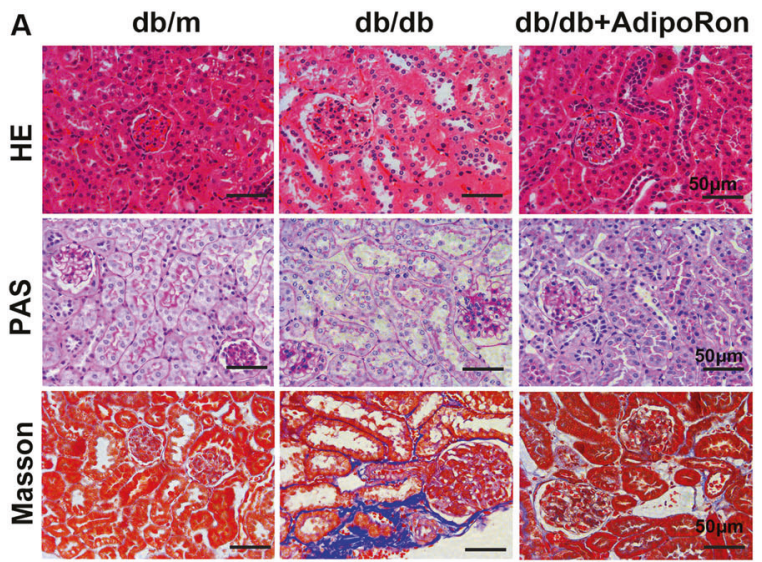

B
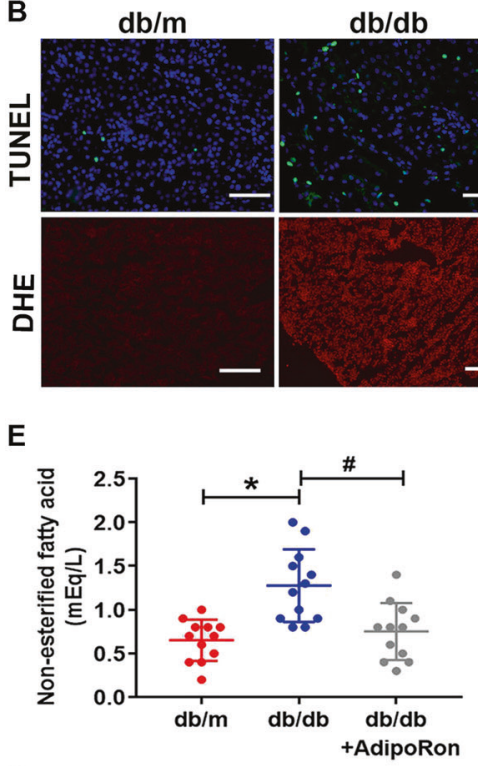

H

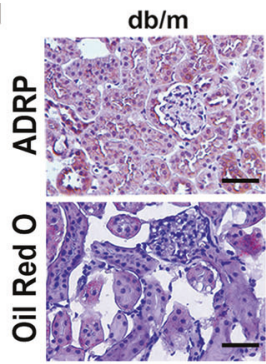

J

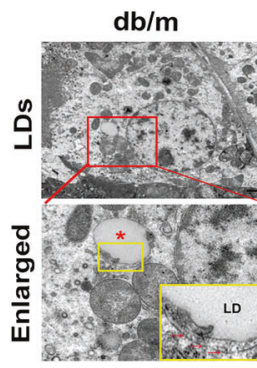

$\mathrm{db} / \mathrm{db}$
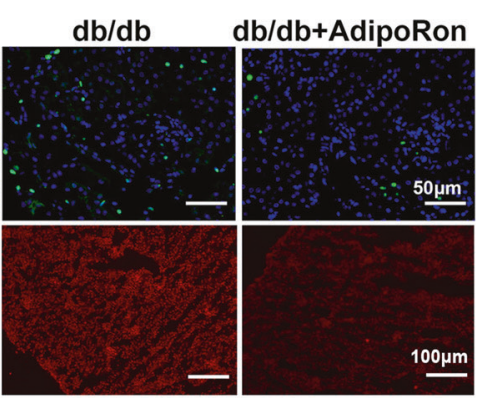

F

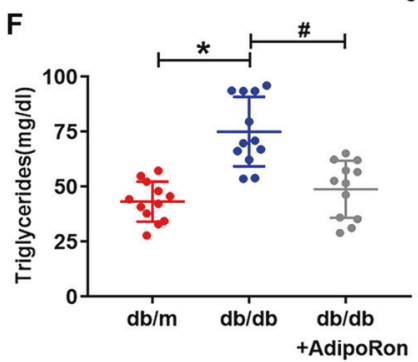

$\mathrm{db} / \mathrm{db}+$ AdipoRon

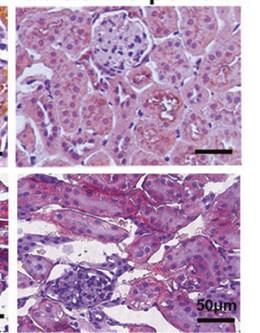

$\mathrm{db} / \mathrm{db}$
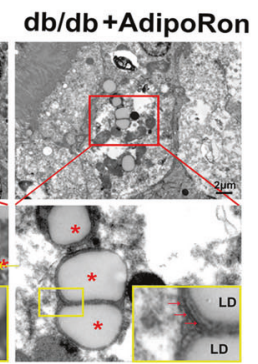

I

K
C

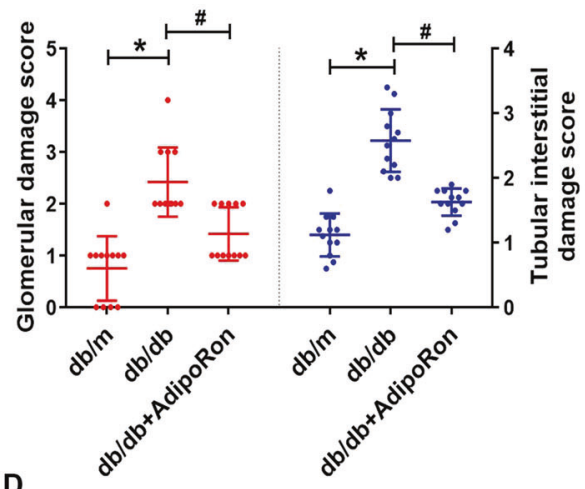

D

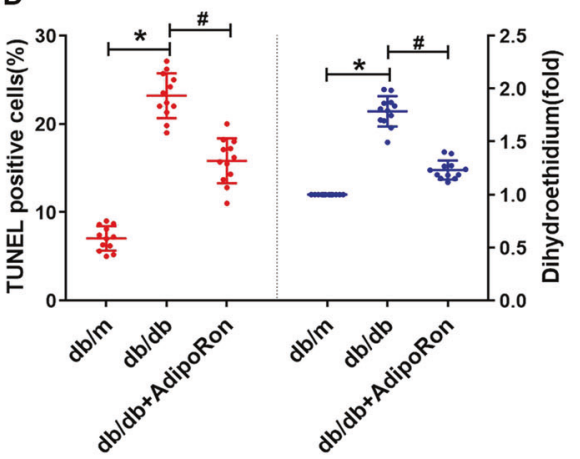

G
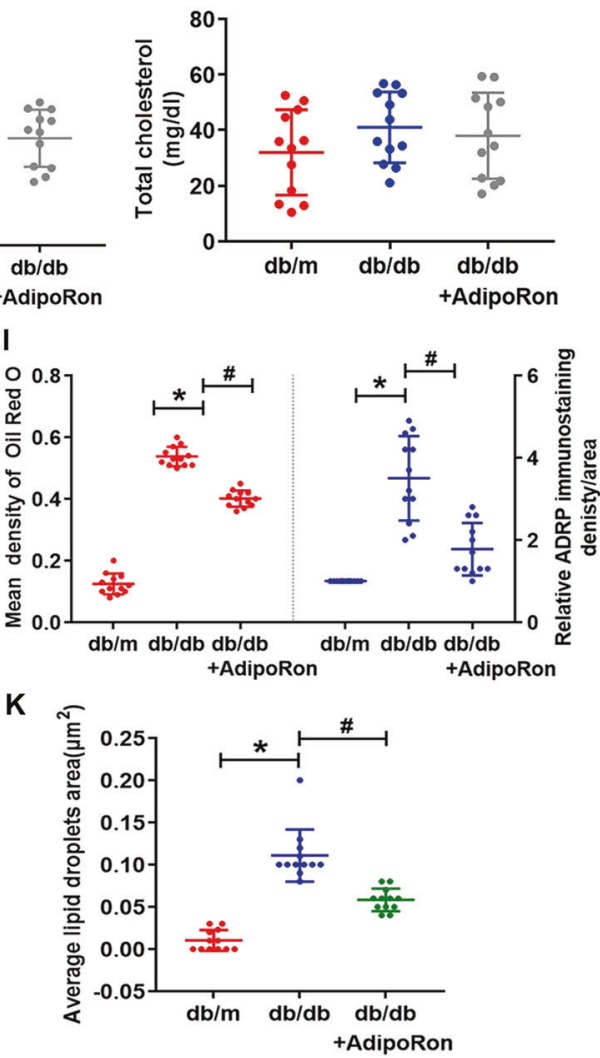

Fig. 3 AdipoRon increases lipophagy, ameliorated lipid deposition and renal pathological injury of db/db mice. Kidney sections stained with HE, PAS, Masson trichrome, TUNEL (magnification, $\times 400)$ and DHE (magnification, $\times 200)(\mathbf{A}$, B). Glomerular damage score and tubular interstitial damage score (C). TUNEL-positive cells in the kidney tissues and quantitative analysis of intrarenal DHE (D). Renal non-esterified fatty acids in the kidney tissues (E). Renal triglycerides (F). Renal total cholesterol (G). IHC analysis of ADRP and Oil-Red O staining in the kidneys (H). Semiquantification of the mean density of Oil-Red O and ADRP expression in the kidney (I). TEM analysis of lipid deposition in the kidney tissues of three groups of mice (magnification, $\times 10,000)$. * indicates the LDs, and red arrows indicate double-membrane autophagic vacuoles containing lipidsnamely, lipophagy (J). Average LD area in the kidney tissues of the three mouse groups (K). Values are the mean \pm $\mathrm{SD} ;{ }^{*} p<0.05$ versus control group; $\# p<0.05$ versus $\mathrm{db} / \mathrm{db}$ group. $n=12$. 
A
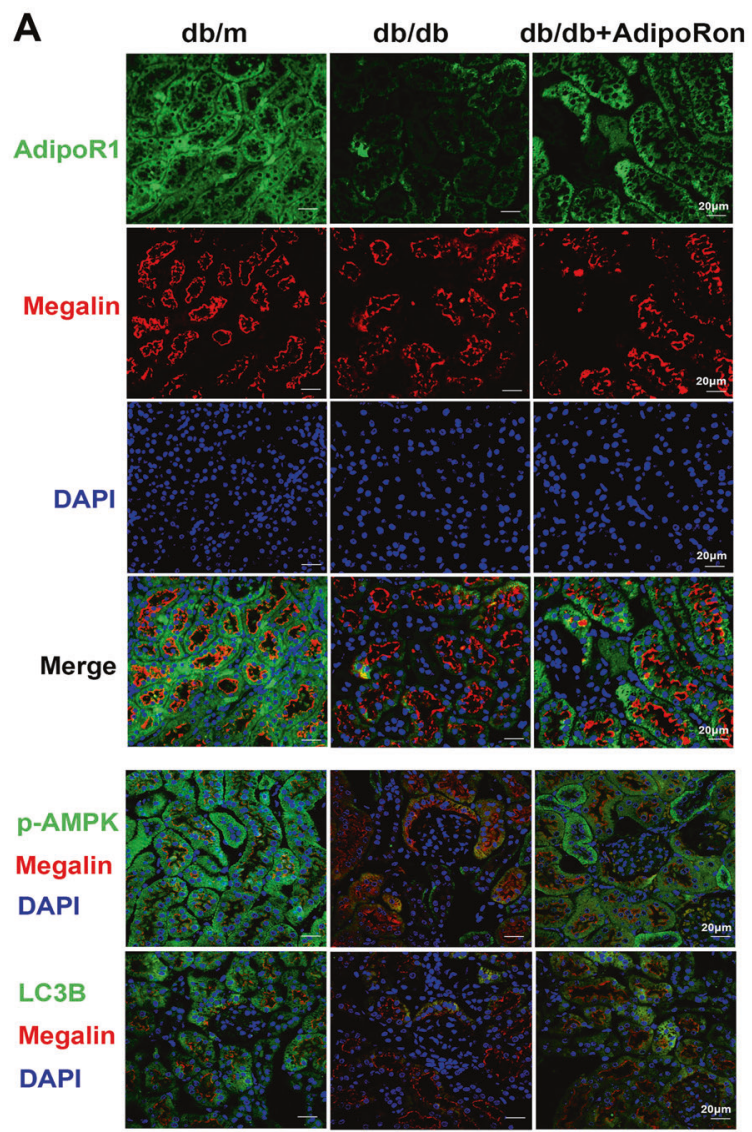

E

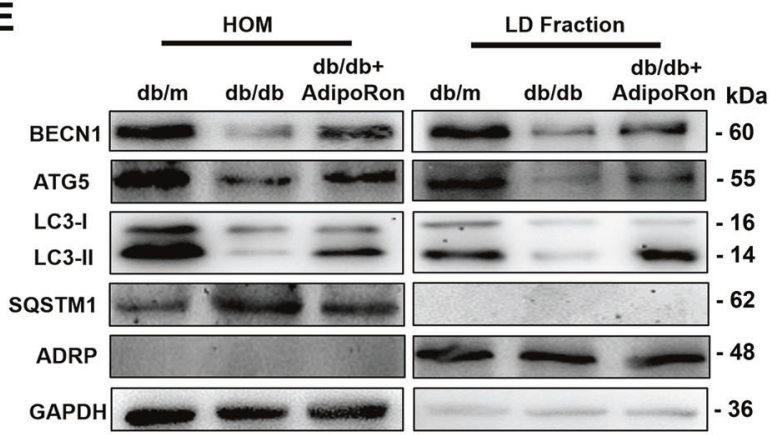

F
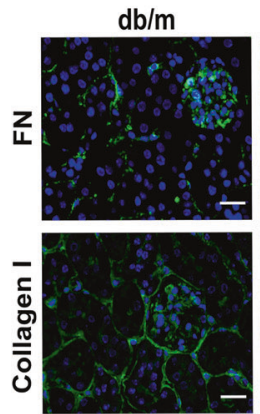

$\mathrm{db} / \mathrm{db}$

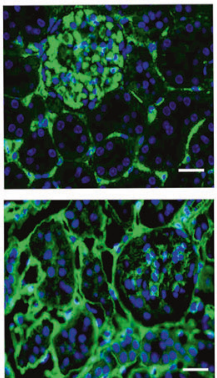

$\mathrm{db} / \mathrm{db}+$ AdipoRon

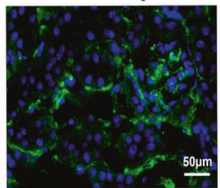

B

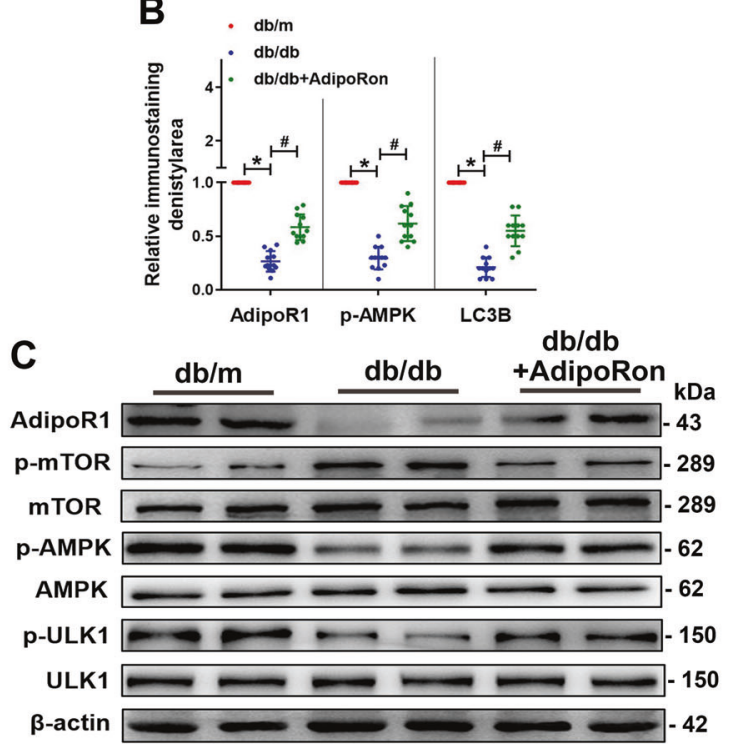

D

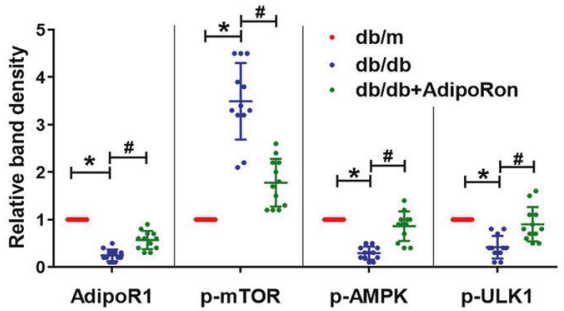

G

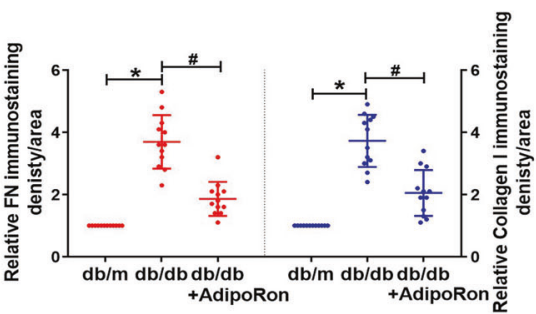

H

$\mathrm{db} / \mathrm{db}$

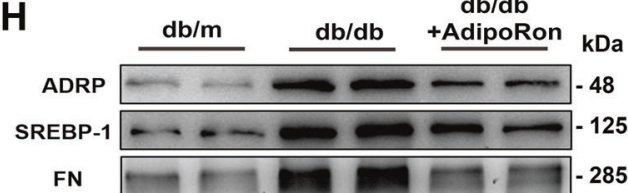

Collagen I $120-130$

$\beta$-actin $\longrightarrow-\infty-\infty-\infty 2$

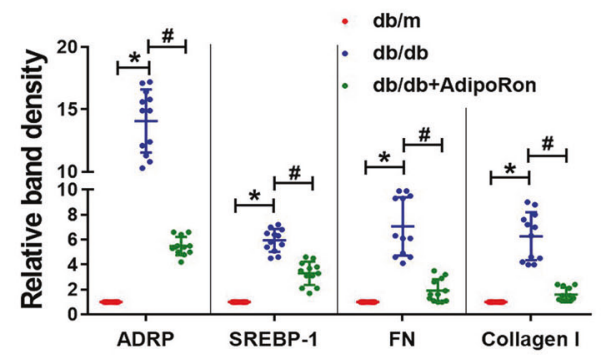

Fig. 4 AdipoRon increased lipophagy, activated AdipoR1/AMPK pathway and alleviated fibrosis in the kidney of db/db mice. Representative IF images of AdipoR1, p-AMPK, and LC3B in the kidney tissues (magnification, $\times 400)($ A). Semiquantification of the IF analysis of AdipoR1, p-AMPK and LC3B (B). WB analysis and quantification of AdipoR1, p-mTOR, p-AMPK and p-ULK1 expression in the kidneys (C, D). WB analysis of the indicated molecules using homogenates (HOM) and isolated LD fractions from the kidneys (E). Representative IF images and semiquantification of FN and Collagen I in the kidneys $(\mathbf{F}, \mathbf{G})$. WB analysis and quantification of the ADRP, SREBP-1, FN and Collagen I levels $(\mathbf{H}$, I). Values are the mean $\pm \mathrm{SD}$; ${ }^{*} p<0.05$ versus control, ${ }^{\#} p<0.05$ versus $\mathrm{db} / \mathrm{db}$ group. $n=12$. 
A

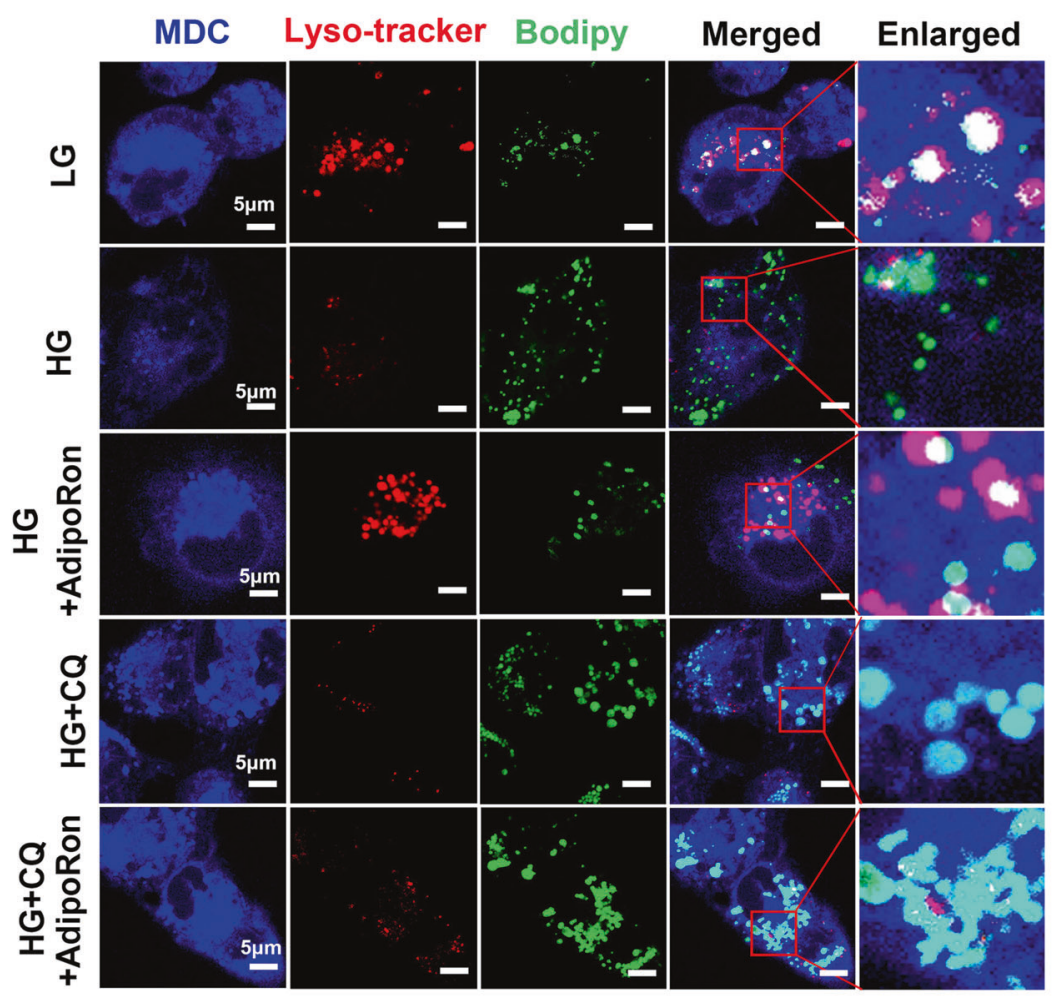

B

LC3 I

LC3 II

ADRP

GAPDH

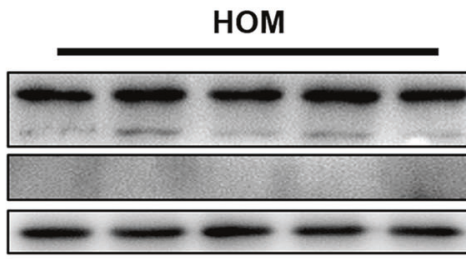

kDa

C
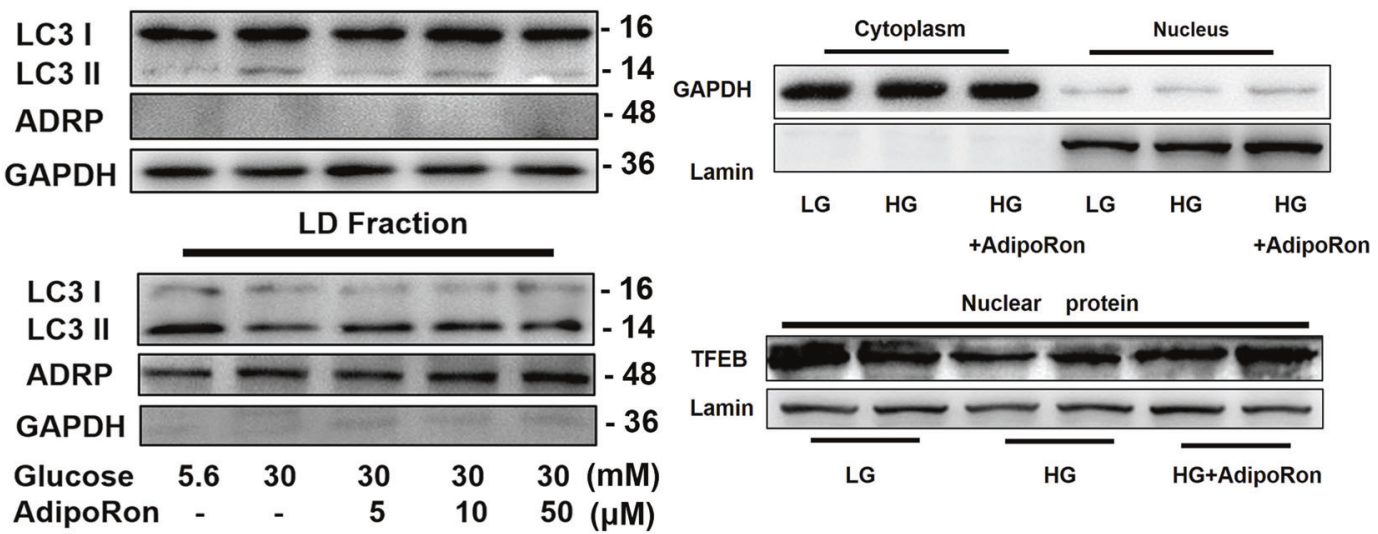

E
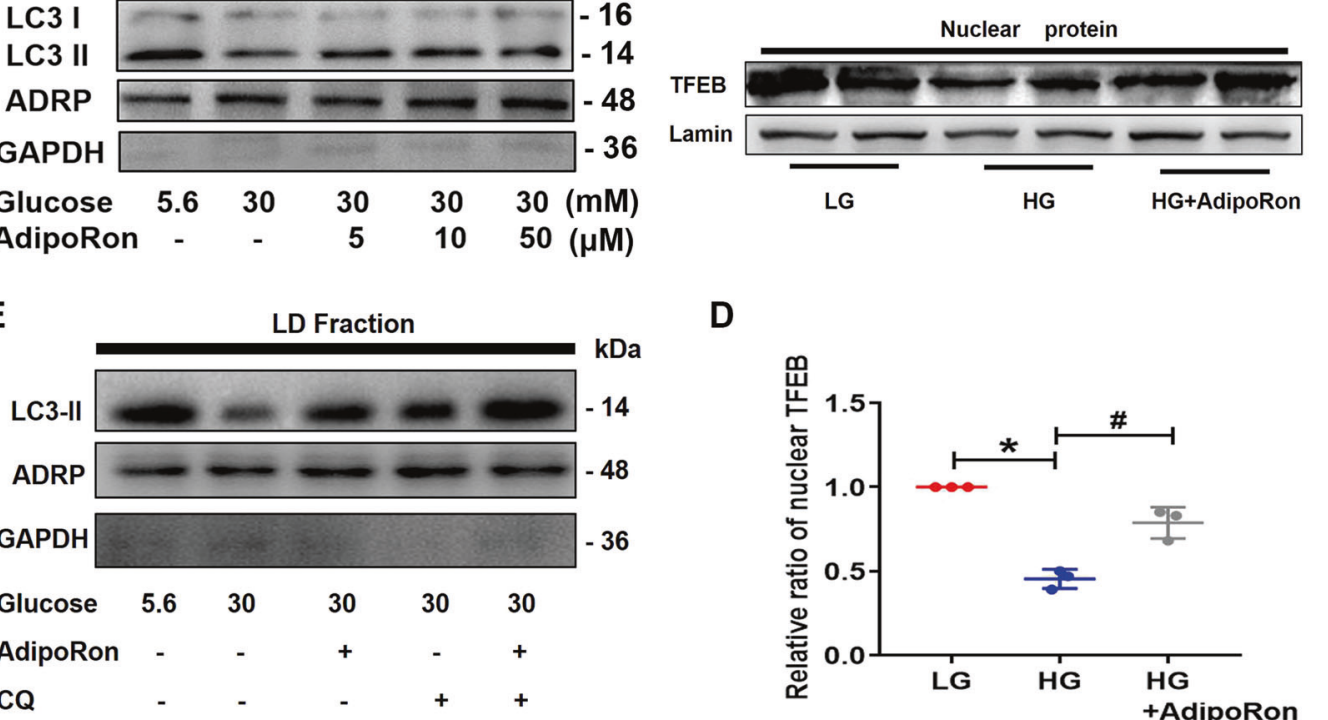

D

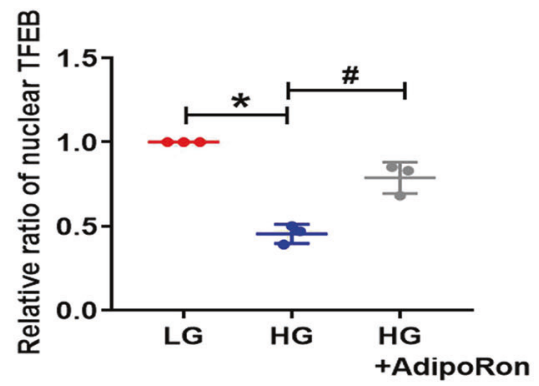

Fig. 5 AdipoRon increased lipophagy in HK-2 cells exposed to HG environment. Representative images of intracellular autophagosome (MCD), lysosomes (Lyso-tracker) and lipid (Bodipy) co-localization in HK-2 cells in an HG environment with or without AdipoRon, CQ pretreatment (A). WB analysis of LC3II expression using HOM and isolated LD fractions from HK-2 cells exposed to HG conditions with or without different AdipoRon concentrations (B). WB and densitometric analysis of TFEB expression using nuclear protein from HK-2 cells exposed to HG conditions with or without AdipoRon (C, D). WB analysis of LC3Il expression using isolated LD fractions from HK-2 cells exposed to HG conditions with or without AdipoRon and CQ pretreatment (E). Values are the mean \pm SD; ${ }^{*} p<0.05$ versus LG group, ${ }^{\#} p<0.05$ versus HG group. $n=3$. 
A

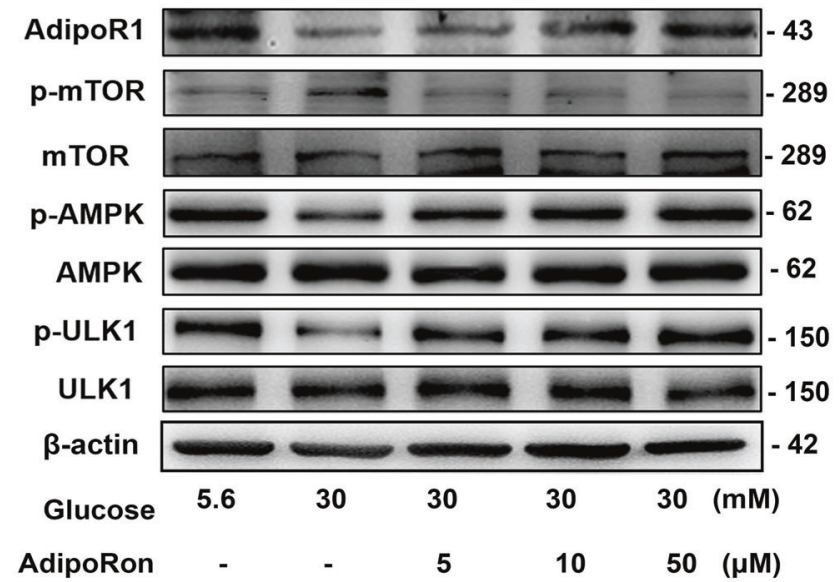

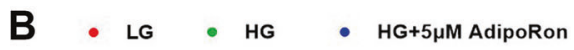

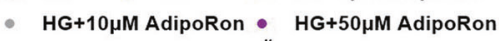

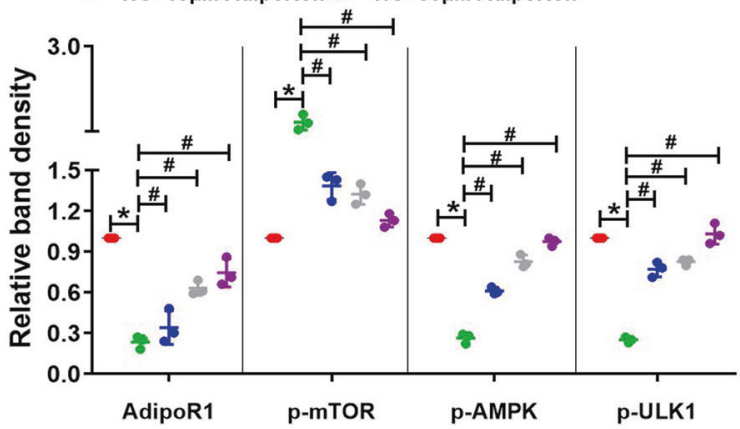

D - HG - HG+AdipoRon - HG+AdipoRon+AdipoR1 siRNA

- HG+AdipoRon+AIACR - HG+AdipoRon+SBI-0206965

- HG+AdipoR1 siRNA+AIACR - HG+AdipoR1 siRNA+SBI-0206965

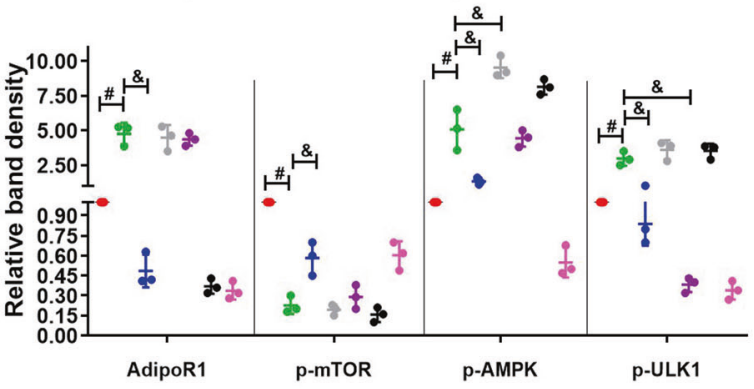

E

BECN1

ATG5

LC3-I

LC3-II

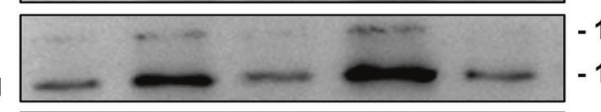

SQSTM1

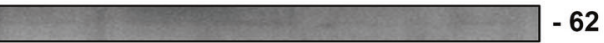

ADRP

GAPDH

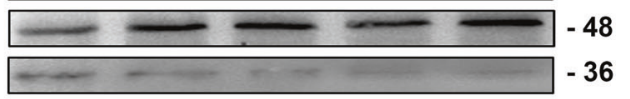

$\begin{array}{llllll}\text { Glucose } & 30 & 30 & 30 & 30 & 30(\mathrm{mM})\end{array}$

AdipoRon - + $+\quad+\quad+$

AdipoR1 siRNA - $\quad-\quad+\quad+\quad-\quad$ -

AICAR

SBI-0206965

Fig. 6 AdipoRon increased lipophagy through AdipoR1/AMPK pathway. WB and densitometric analysis of AdipoR1, p-mTOR, p-AMPK and p-ULK1 expression in HK-2 cells exposed to HG conditions with or without different AdipoRon concentrations (A, B). WB and densitometric analysis of AdipoR1, p-mTOR, p-AMPK and p-ULK1 expression in HK-2 cells exposed to an HG environment pretreated with AdipoRon, AdipoR1 siRNA, AICAR or SBI-0206965 (C, D). WB analysis of BECN1, ATG5, LC3 and SQSTM1 using isolated LD fractions from HK-2 cells exposed to HG conditions with or without AdipoRon, AdipoR1 siRNA, AICAR and SBI-0206965 (E). Values are the mean \pm SD; ${ }^{*} p<0.05$ versus LG group, ${ }^{\#} p<0.05$ versus HG group, ${ }^{\&} p<0.05$ versus HG + AdipoRon group. $n=3$.

the alleviation of renal pathological injury, oxidative stress, apoptosis and fibrosis (Table 2 and Figs. 3 and 4). These data further demonstrated that pharmacological enhancement of the level of autophagy-mediated lipophagy could reverse the ELD and tubular cells injury in DN.

It is reported that AdipoR1 activation by AdipoRon could restore AMPK-mediated autophagosome formation and stimulate autophagosome clearance under diabetic conditions [36]. Additionally, the decreased AdipoR1 and AMPK activation led to a reduction in fatty acid oxidation and an increase in fatty acid synthesis, promoting the progression of type 2 diabetes [37, 38]. The increased AMPK phosphorylation and subsequently activated autophagy has been demonstrated to be a renal protection mechanism in DN $[39,40]$. AMPK is a key enzyme that regulates autophagy, which is involved in the development of DN [41]. Furthermore, it is known that autophagy was promoted by AMPK through directly activating ULK1 phosphorylation, while inhibited by mTOR through disrupting the interaction between ULK1 and AMPK [42]. Additional, ULK1 plays an important role in regulating lipid metabolism in adipocytes by regulating autophagy [43]. Increased levels of p-AMPK and ULK1 were suggested to be related to the activation of lipophagy in hypothalamic neurons [44]. As expected, treatment of HK-2 cells with AdipoRon reversed HG-induced lipophagy dysfunction and increased lipid deposition, while these effects were partially blocked by pretreatment with autophagy inhibitor, but enhanced by AMPK activtor (Figs. 5-7). 

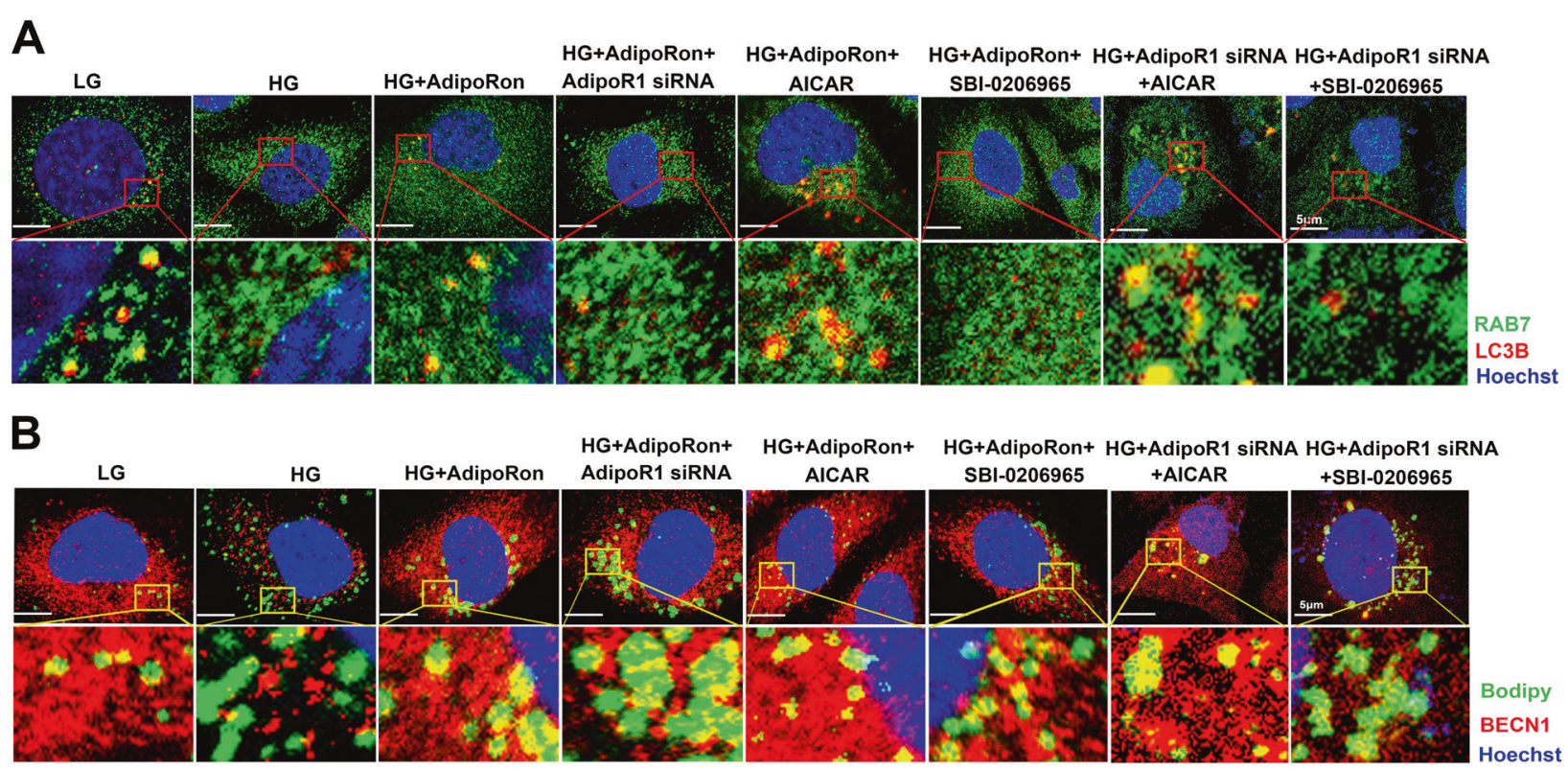

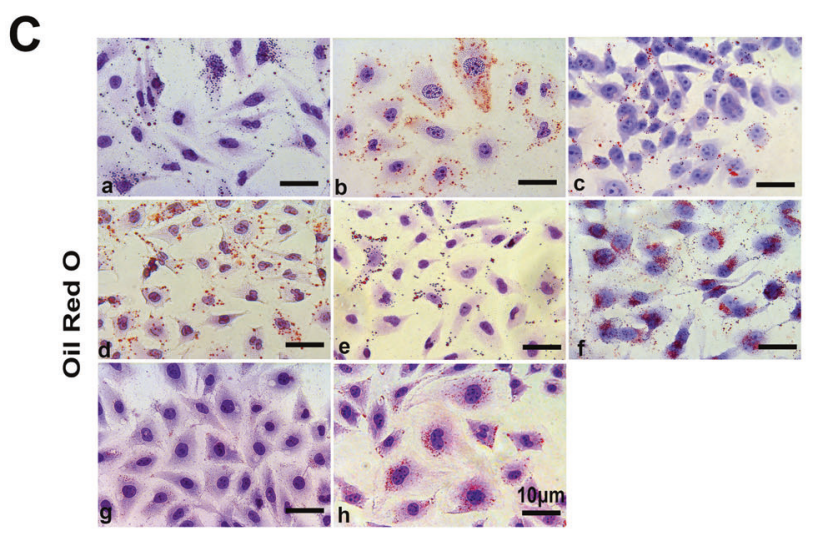

a. LG b. HG c. HG+AdipoRon d. HG+AdipoRon+AdipoR1 siRNA e. HG+AdipoRon+AICAR f. HG+AdipoRon+SBI-0206965 g. HG+AdipoR1 siRNA+AICAR h. HG+AdipoR1 siRNA+SBI-0206965

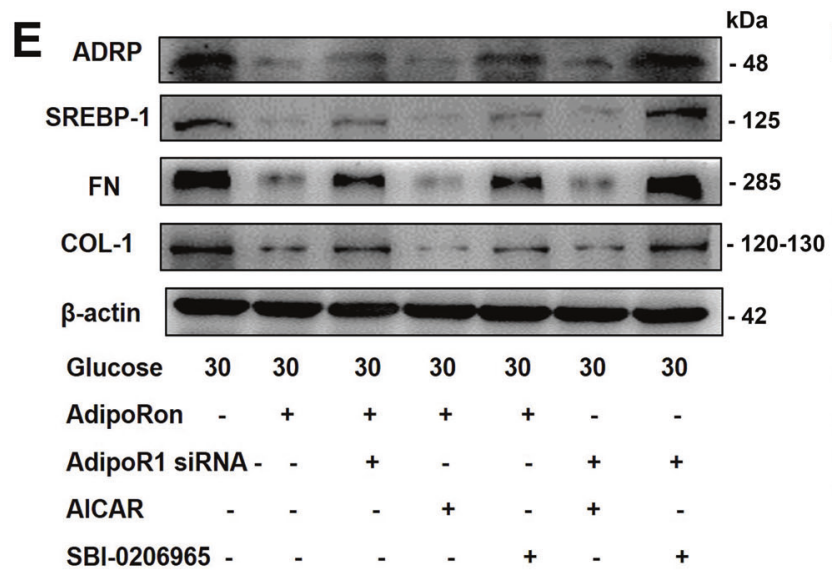

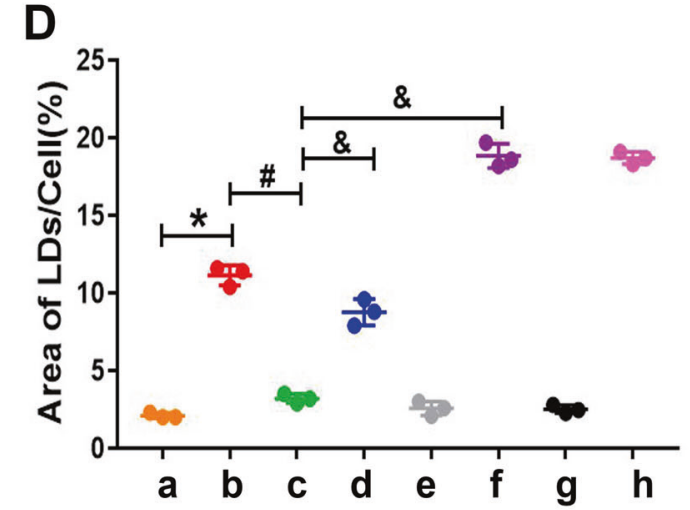

a. LG b. HG c. HG+AdipoRon d. HG+AdipoRon+AdipoR1 siRNA e. HG+AdipoRon+AICAR f. HG+AdipoRon+SBI-0206965 g. HG+AdipoR1 siRNA+AICAR h. HG+AdipoR1 siRNA+SBI-0206965
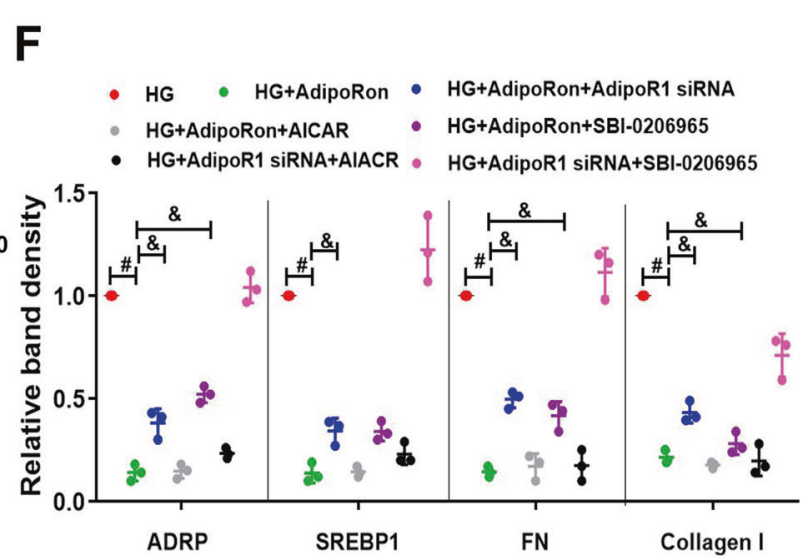

Fig. 7 The effects of AdipoRon on increasing lipohagy and alleviating lipid deposition and fibrosis were partially blocked by ULK1 inhibitor, while enhance by AMPK activator. Confocal IF images showing the co-localization of RAB7 and LC3B and the co-localization of BECN1 and LDs (Bodipy) in HK-2 cells exposed to HG conditions and pretreated with or without AdipoRon, AdipoR1 siRNA, AICAR and SBI0206965 (A, B). Representative images and semiquantification of the lipid deposition in HK-2 cells exposed to an HG environment pretreated with AdipoRon, AdipoR1 siRNA, AICAR and SBI-0206965 (C, D). WB and densitometric analysis of ADRP, SREBP-1, FN and Collagen I expressions in HK-2 cells exposed to HG conditions with or without AdipoRon, AdipoR1 siRNA, AICAR and SBI-0206965 (E, F). Values are the mean \pm SD; * $p$ $<0.05$ versus LG group, ${ }^{\#} p<0.05$ versus HG group. $n=3$. 
These data suggested that increased lipophagy by AdipoRon in tubular cells of DN might through activating AdipoR1/AMPK pathway.

Briefly, in this study we found that the lipophagy deficiency plays a critical role in the ELD and lipotoxicity in tubular cells of DN. AdipoRon can reduce intrarenal lipotoxicity-associated renal injury and fibrosis in DN through increasing lipophagy by activating AdipoR1/AMPK pathway. These findings suggest that targeting lipophagy might be a potential therapeutic strategy for DN and other ELD-related metabolic diseases.

\section{MATERIALS AND METHODS \\ Antibodies and reagents}

We used the following commercially available antibodies: The antiAdipoR1 (ab126611), anti-FN (ab2413), anti-Collagen I (ab34710), antiAMPKa1 + AMPKa2 (ab131512), anti-AMPK alpha 1 (phospho T183) + AMPK alpha 2 (phospho T172) (ab23875), anti-RAB7(ab50533) and antiADRP (ab52356) antibodies were from Abcam; the anti-phospho-AMPKa (Thr172) (2535), anti-mTOR(2972), anti-phospho-mTOR(Ser2448)(5536), anti-ULK1 (8054), anti-phospho-ULK1 (Ser555) (5869), anti-SQSTM1/p62 (5114) and anti-LC3B (3868) antibodies were from Cell Signaling Technology; and the anti-SQSTM1/P62 (18420-1-AP), anti-LC3B (187251-AP), anti-Beclin1 (11306-1-AP), anti-ADRP (15294-1-AP), anti-ATG5 (10181-2-AP), anti-TFEB (13372-1-AP), anti- $\beta$-actin (60008-1-lg), antiGAPDH (60004-1-lg) and anti-Lamin B(12987-1-AP) antibodies were from Proteintech. The anti-SREBP-1c(sc-13551) was from Santa Cruz Biotechnology. AdipoRon and CQ was obtained from Selleckchem. AICAR was provided by Apexbio Technology, SBI-0206965 was purchased from Sigma-Aldrich.

\section{Clinical data}

Patients with DN were newly diagnosed by renal biopsy who used insulin to control blood glucose. Patients using lipid-lowering drugs, RAS inhibitors before biopsy and those with urinary tract infections, or with inflammatory, neoplastic, cardiovascular, hepatic, renal, lung or neuroendocrine diseases were excluded. The patients in the control group were the first diagnosed patients with primary glomerulonephritis of varying degrees of proteinuria and the pathological diagnosis was minimal change nephropathy. These patients were not treated prior to pathological diagnosis. The DN group included 15 patients $(8$ males and 7 females), the average age is $43.69 \pm 7.62$ years (34-57 years) and the control group included 15 patients ( 8 males and 7 females), the average age is $41.54 \pm 7.66$ years (32-56 years). The clinical experimental procedures were approved by the Ethics Committee of Second Xiangya Hospital, Central South University, and informed consent was obtained from all the patients.

\section{Animal experimental design}

Fourteen-week-old male C57BLKS/J db/db and C57BLKS/J db/m mice were purchased from the Aier Matt Experimental Animal Company (China). They were randomly divided into the following three groups: a $\mathrm{db} / \mathrm{m}$ group (control, $n=12$ ), a $\mathrm{db} / \mathrm{db}$ group receiving intragastric injection with AdipoRon $(n=12)$ and a $\mathrm{db} / \mathrm{db}$ group that receive intragastric injection with $0.5 \%$ sodium carboxymethyl cellulose solution, which served as vehicle control $(n=12)$. AdipoRon $(30 \mathrm{mg} /$ $\mathrm{kg}$ ) was dissolved in $0.5 \%$ sodium carboxymethyl cellulose solution and then provided to $\mathrm{db} / \mathrm{db}$ mice once daily via oral gavage from 16 weeks of age for 4 weeks as described previously [45]. The mice were euthanized at 20 weeks of age. All animal procedures were approved by The Animal Care and Use Committee of Second Xiangya Hospital of Central South University.

\section{Assessment of physiological features}

Body weights and blood glucose levels were measured twice per week. Twenty-four-hour urine samples were collected using metabolic cages before the mice were euthanized. Urine albumin concentrations were tested using an Albuwell $M$ kit, and levels of serum creatinine were measured using HPLC as previous study [45]. The intrarenal lipid levels, namely FFAs, TG and TC using commercial kits (Wako, Osaka, Japan) according to manufacturer's instructions.

\section{Morphological analysis}

Renal tissues were fixed in $4 \%$ paraformaldehyde (PFA) and then embedded in paraffin. Four-micrometer thick paraffin-embedded tissue sections were prepared and subjected to hematoxylin-eosin (HE), periodic acid-Schiff (PAS), periodic acid-silver methe-namine (PASM) and Masson trichrome staining as previously described [2]. Tubulo-interstitial lesion and glomerular injury indices were evaluated as described previously $[46,47]$.

\section{IHC of renal tissue}

Four-micrometer -thick paraffin-embedded renal tissue sections from human or mice were prepared for IHC studies, as previously described [3]. The sections were deparaffined in xylene, rehydrated in ethanol and soaked in $10 \mathrm{mM}$ citrate buffer at $65^{\circ} \mathrm{C}$ for antigen retrieval. After blocking endogenous peroxidase activity with $3 \% \mathrm{H}_{2} \mathrm{O}_{2}$, the sections were exposed to $5 \%$ bovine serum albumin (BSA) and sequentially incubated with antibodies against ADRP $\left(1: 100\right.$, Proteintech, 15294-1-AP) overnight at $4{ }^{\circ} \mathrm{C}$. The sections were then incubated with secondary antibodies conjugated with peroxidase, treated with diaminobenzidine, and finally counterstained with hematoxylin. Images were obtained using a Nikon microscope and analyzed by ImageJ.

\section{IF, apoptosis assay, and ROS accumulation determination of renal tissue}

We performed IF analysis of AdipoR1(1:100, Abcam, ab126611), p-AMPK (1:200, Abcam, ab23875), LC3B (1:100, Proteintech, 18725-1-AP), FN (1:200, Abcam, ab2413), and Collagen I (1:200, Abcam, ab34710) by using 4\% PFAfixed, paraffin-embedded renal tissue sections ( $4 \mu \mathrm{m}$ thick). Apoptotic renal cells were detected using the TUNEL assay with an In-Situ Cell Death Detection Kit (Roche Applied Science, China), in accordance with the manufacturer's instructions. ROS in renal tissues were determined by oxidative fluorescent dye dihydroethidine (DHE, $1 \mu \mathrm{M}$, Invitrogen).

\section{WB analysis}

RIPA buffer (CWBIO, China) supplemented with protease inhibitors and phosphatase inhibitors (CWBIO, China) was used to extracted protein from kidney tissues or HK-2 cells. Protein concentration was quantified by the BCA method. Equal amounts of protein were then analyzed by sodium dodecyl sulphate-polyacrylamide gel electrophoresis. Primary antibodies specific for AdipoR1 (1:3000, Abcam, ab126611), phospho-AMPKa(Thr172) (1:1000, Cell Signaling Technology, 2535), AMPKa1 + AMPKa2 (1:2000, Abcam, ab131512), phospho-ULK1 (Ser555) (1:1000, Cell Signaling Technology, 5869), ULK1 (1:1000, Cell Signaling Technology, 8054), ADRP (1:500, Abcam, ab52356), FN (1:1000, Abcam, ab2413), Collagen I (1:1000, Abcam, ab34710), LC3B (1:1000, Cell Signaling Technology, 3868), Beclin1 (1:800, Proteintech, 11306-1-AP), ATG5 (1:1000, Proteintech, 10181-2-AP), SQSTM1/ P62 (1:1000, Proteintech, 18420-1-AP, Cell Signaling Technology, $1: 1000,5114)$ and TFEB $(1: 1000$, Proteintech, 13372-1-AP) were used. The bands were evaluated using a Tanon 5200 Multi instrument (Tanon Instruments, China). The band densities of target proteins were compared with those of $\beta$-actin (1:5000, Proteintech, 60008-1-lg), GAPDH (1:5000, Proteintech, $60004-1-\mathrm{lg})$ or Lamin B $(1: 1000$, Proteintech, $66095-1-\mathrm{lg})$ by using densitometry software (ImageJ).

\section{Cell culture and treatments}

HK-2 cells were cultured as previously described [2]. Briefly, HK-2 cells were exposed to different concentrations of D-glucose $(5.6 \mathrm{mM}$ or $30 \mathrm{mM})$, with or without AdipoRon $(5 \mathrm{nM}, 10 \mathrm{nM}$, and $50 \mathrm{nM})$, AICAR $(0.5 \mathrm{mM}), \mathrm{SBI}-$ $0206965(10 \mu \mathrm{m})$ or CQ $(50 \mu \mathrm{m})$. For gene disruption, HK-2 cells were pretransfected with AdipoR1 siRNA using Lipofectamine 3000 (Invitrogen, USA) in accordance with the manufacturer's protocol.

\section{Lipophagy analysis}

Lipophagy was monitored with the following methods. (1) Oil-Red $O$ staining: Five-micrometer-thick, 4\% PFA-fixed, frozen renal tissue sections or $4 \%$ PFA-fixed HK-2 cells were rinsed with $60 \%$ isopropanol for 20-30 seconds, and stained with Oil-Red $O$ dye solution for $15 \mathrm{~min}$ as previously described [12]. (2) TEM: Kidney samples were fixed in $2.5 \%$ glutaraldehyde in $0.1 \mathrm{M}$ phosphate buffer $\left(\mathrm{pH} \mathrm{7.4)}\right.$ ) overnight at $4{ }^{\circ} \mathrm{C}$ and fixed with $1 \%$ osmium tetroxide solution for $1 \mathrm{~h}$. The samples were then dehydrated in ethanol and embedded in Epon 812. Ultrathin sections $(\sim 80 \mathrm{~nm})$ were prepared and stained with uranyl acetate and lead citrate, 
followed by observation of the LD-associated double-membrane structure using TEM. (3) WB analysis: Lipid droplet components were isolated from mouse kidney samples and HK-2 cells as previously described [48]. Briefly, kidney samples from 8 kidneys were homogenized by ultrasonic disruption after being kept in icy buffer A ( $20 \mathrm{mM}$ tricine, $250 \mathrm{mM}$ sucrose, $\mathrm{PH}=7.8$ ) plus $0.2 \mathrm{mM}$ phenyl methane sulfonyl fluoride (PMSF) for $20 \mathrm{~min}$. Then, the samples were centrifuged at $100 \times g$ for $10 \mathrm{~min}$ at $4{ }^{\circ} \mathrm{C}$, and the supernatant was disrupted under high pressure. The homogenate was centrifuged at $3000 \times g$ for $10 \mathrm{~min}$ at $4{ }^{\circ} \mathrm{C}$ to obtain the postnuclear supernatant (PNS) fraction. Ten milliliters of PNS and two milliliters of buffer B ( $20 \mathrm{mM}$ HEPES, $100 \mathrm{mM} \mathrm{KCl}$ and $2 \mathrm{mM} \mathrm{MgCl} 2, \mathrm{pH}=7.4$ ) were placed into a SW40 tube and centrifuged by an ultracentrifuge at $100,000 \times g$ for $60 \mathrm{~min}$ at $4{ }^{\circ} \mathrm{C}$. The crude LD fraction was carefully collected from the top to the Eppendorf tube. Finally, the crude LD fraction was resuspended in $200 \mu \mathrm{L}$ of buffer $\mathrm{B}$ in the Eppendorf tube and centrifuged at $20,000 \times g$ for $5 \mathrm{~min}$ at $4{ }^{\circ} \mathrm{C}$ twice to purify the LD fraction. For HK-2 cells, cells were washed with ice-cold PBS before collection, followed by centrifugation at $1000 \times g$ for $10 \mathrm{~min}$ at $4^{\circ} \mathrm{C}$. The samples were then suspended in buffer A plus $0.2 \mathrm{mM}$ PMSF and incubated on ice for $20 \mathrm{~min}$, followed by ultrasonic disruption. After centrifugation at $3000 \times g$ for $10 \mathrm{~min}$ at $4{ }^{\circ} \mathrm{C}$, and the PNS fraction and buffer $\mathrm{B}$ were transferred into a SW40 tube to centrifuge at $182,000 \times g$ for $60 \mathrm{~min}$ at $4^{\circ} \mathrm{C}$. The LD fraction was carefully collected from the top band of the gradient and sequentially washed and purified three times by buffer $B$ as mentioned above. Finally, an equal amount of the LD protein was analyzed by WB analysis. (4) Confocal microscopy: Confocal microscopy was carried out to detect the colocalization of LDs (BODIPY 493/503 neutral lipid stains), autophagosomes (MDC stains) and lysosomes (Lyso-tracker stains) the co-localization of RAB7 and LC3B and the co-localization of BECN1 and LDs (BODIPY 493/503 neutral lipid stains) in HK-2 cells. The analysis was performed using LSM 510 software (Zeiss).

\section{Cell IF}

Treated HK-2 cells were fixed with 4\% PFA for 5 min, permeabilized with ice-cold methanol for $10 \mathrm{~min}$ at $-20^{\circ} \mathrm{C}$, blocked with blocking buffer (PBS $+1 \% \mathrm{BSA}+0.3 \%$ TritonX-100) for $1 \mathrm{~h}$ at $22^{\circ} \mathrm{C}$, and then incubated with primary antibodies against RAB7 (1:2000, Abcam, ab50533), LC3B (1:100, Proteintech, 18725-1-AP) and Beclin1 (1:100, Proteintech, 11306-1-AP) for $2 \mathrm{~h}$ at $22^{\circ} \mathrm{C}$. After washing with PBS, Alexa Fluor ${ }^{\circledR}$ 594-conjugated goat anti-rabbit or Alexa Fluor ${ }^{\circledR}$ 488-conjugated goat anti-mouse antibodies were applied for $1 \mathrm{~h}$ at $37^{\circ} \mathrm{C}$, followed by counterstaining with Hoechst to delineate the nuclei. Cells were then examined by LSM 780 META laser scanning microscopy.

\section{Extraction of nuclear protein}

Separation of nuclear protein from the cytoplasmic protein was carried out using the Nuclear and Cytoplasmic Protein Extraction Kit (Beyotime) according to the manufacturer's instructions. Briefly, HK-2 cells were resuspended in ice-cold hypotonic lysis buffer after intervention, and subcellular protein fractions extracted by homogenizing and centrifuging. Finally, an equal amount of the nuclear protein was analyzed by WB analysis. Primary antibodies specific for TFEB (1:1000, Proteintech, 13372-1AP) were used. The bands were evaluated using a Tanon 5200 Multi instrument (Tanon Instruments, China). The band densities of target proteins were compared with those of Lamin B (1:1000, Proteintech, 66095-1-lg) by using ImageJ.

\section{Bioinformatics and Nephroseq analysis}

Normal human renal RNAseq data were obtained from Genotype-Tissue Expression (GTEx) for AdipoR1 gene coexpression analysis, genes correlated with Pearson $r>0.6$ and $p<0.05$ were subjected to GO analysis via DAVID online tools (Version 6.8). Data for the analysis of the correlation between the gene expression of AdipoR1 and renal function index (eGFR and Scr) were obtained by the publicly available data sets from the Nephroseq database (https://www.nephroseq.org/resource/login.html, V5).

\section{Statistical analysis}

All statistical analysis was performed using the SPSS version 17.0 (SPSS Inc., Chicago, IL, USA) and GraphPad Prism 7.0 (GraphPad Software Inc., San Diego, (A, USA). The experimental data are expressed as the mean \pm standard deviation. Differences between two groups were tested using $t$ test or Mann-Whitney U-test. For more than two groups, one-way analysis of variance (ANOVA) with Tukey's post hoc analysis was used. The correlation between two variables was tested by Pearson's correlation analysis. $p<0.05$ was considered statistically significant.

\section{DATA AVAILABILITY}

All data generated or analyzed during this study are included in this published article.

\section{REFERENCES}

1. Zhang L, Long J, Jiang W, Shi Y, He X, Zhou Z, et al. Trends in chronic kidney disease in China. N Engl J Med. 2016;375:905-6.

2. Han Y, Xu X, Tang C, Gao P, Chen X, Xiong X, et al. Reactive oxygen species promote tubular injury in diabetic nephropathy: The role of the mitochondrial ros-txnip-nlrp3 biological axis. Redox Biol. 2018;16:32-46.

3. Xiao L, Xu X, Zhang F, Wang M, Xu Y, Tang D, et al. The mitochondria-targeted antioxidant MitoQ ameliorated tubular injury mediated by mitophagy in diabetic kidney disease via Nrf2/PINK1. Redox Biol. 2017;11:297-311.

4. Herman-Edelstein M, Scherzer P, Tobar A, Levi M, Gafter U. Altered renal lipid metabolism and renal lipid accumulation in human diabetic nephropathy. J Lipid Res. 2014:55:561-72.

5. Stadler K, Goldberg IJ, Susztak K. The evolving understanding of the contribution of lipid metabolism to diabetic kidney disease. Curr Diab Rep. 2015;15:40.

6. Engin AB. What Is Lipotoxicity? Adv Exp Med Biol. 2017;960:197-220.

7. Estadella D, da Penha Oller do Nascimento CM, Oyama LM, Ribeiro EB, Damaso AR, de Piano A. Lipotoxicity: effects of dietary saturated and transfatty acids. Mediators Inflamm. 2013;2013:137579.

8. Opazo-Rios L, Mas S, Marin-Royo G, Mezzano S, Gomez-Guerrero C, Moreno JA. et al. Lipotoxicity and diabetic nephropathy: novel mechanistic insights and therapeutic opportunities. Int J Mol Sci. 2020;21:2632.

9. Zhao X, Chen X, Zhang Y, George J, Cobbs A, Wang G. et al. Kidney injury molecule-1 is upregulated in renal lipotoxicity and mediates palmitate-induced tubular cell injury and inflammatory response. Int J Mol Sci. 2019;20:3406.

10. Choi SR, Lim JH, Kim MY, Kim EN, Kim Y, Choi BS, et al. Adiponectin receptor agonist AdipoRon decreased ceramide, and lipotoxicity, and ameliorated diabetic nephropathy. Metabolism. 2018;85:348-60.

11. Yamamoto $T$, Takabatake $Y$, Takahashi A, Kimura T, Namba T, Matsuda J, et al. High-fat diet-induced lysosomal dysfunction and impaired autophagic flux contribute to lipotoxicity in the kidney. J Am Soc Nephrol. 2017;28:1534-51.

12. Chen $X$, Han $Y$, Gao $P$, Yang $M$, Xiao $L$, Xiong $X$, et al. Disulfide-bond $A$ oxidoreductase-like protein protects against ectopic fat deposition and lipidrelated kidney damage in diabetic nephropathy. Kidney Int. 2019;95:880-95.

13. Amir M, Czaja MJ. Autophagy in nonalcoholic steatohepatitis. Expert Rev Gastroenterol Hepatol. 2011;5:159-66.

14. Ouimet M, Franklin V, Mak E, Liao X, Tabas I, Marcel YL. Autophagy regulates cholesterol efflux from macrophage foam cells via lysosomal acid lipase. Cell Metab. 2011;13:655-67.

15. Singh R, Kaushik S, Wang Y, Xiang Y, Novak I, Komatsu M, et al. Autophagy regulates lipid metabolism. Nature. 2009;458:1131-5.

16. Jaishy B, Abel ED. Lipids, lysosomes, and autophagy. J Lipid Res. 2016;57:1619-35.

17. Ward C, Martinez-Lopez N, Otten EG, Carroll B, Maetzel D, Singh R, et al. Autophagy, lipophagy and lysosomal lipid storage disorders. Biochim Biophys Acta. 2016;1861:269-84.

18. Singh R, Cuervo AM. Lipophagy: connecting autophagy and lipid metabolism. Int J Cell Biol. 2012;2012:282041.

19. Ding WX, Li M, Chen X, Ni HM, Lin CW, Gao W, et al. Autophagy reduces acute ethanol-induced hepatotoxicity and steatosis in mice. Gastroenterology. 2010;139:1740-52.

20. Kovsan J, Bluher M, Tarnovscki T, Kloting N, Kirshtein B, Madar L, et al. Altered autophagy in human adipose tissues in obesity. J Clin Endocrinol Metab. 2011;96: E268-77.

21. Straniero S, Cavallini G, Donati A, Pallottini V, Martini C, Trentalance A, et al. Stimulation of autophagy by antilipolytic drugs may rescue rodents from ageassociated hypercholesterolemia. Rejuvenation Res. 2009;12:77-84.

22. Zhou $\mathrm{K}$, Yao $\mathrm{P}$, He J, Zhao $\mathrm{H}$. Lipophagy in nonliver tissues and some related diseases: pathogenic and therapeutic implications. J Cell Physiol. 2019;234:7938-47.

23. Xiong J, Wang K, He J, Zhang G, Zhang D, Chen F. TFE3 Alleviates hepatic steatosis through autophagy-induced lipophagy and PGC1alpha-mediated fatty acid beta-oxidation. Int J Mol Sci. 2016;17:387.

24. Carmona-Gutierrez D, Zimmermann A, Madeo F. A molecular mechanism for lipophagy regulation in the liver. Hepatology. 2015;61:1781-3.

25. Yamahara K, Kume S, Koya D, Tanaka Y, Morita Y, Chin-Kanasaki M, et al. Obesitymediated autophagy insufficiency exacerbates proteinuria-induced tubulointerstitial lesions. J Am Soc Nephrol. 2013;24:1769-81. 
26. Kounakis K, Chaniotakis M, Markaki M, Tavernarakis N. Emerging Roles of lipophagy in health and disease. Front Cell Dev Biol. 2019;7:185.

27. de Vries AP, Ruggenenti $P$, Ruan XZ, Praga M, Cruzado JM, Bajema IM, et al. Fatty kidney: emerging role of ectopic lipid in obesity-related renal disease. Lancet Diabetes Endocrinol. 2014;2:417-26.

28. Martinez-Garcia C, Izquierdo-Lahuerta A, Vivas Y, Velasco I, Yeo TK, Chen S, et al. Renal lipotoxicity-associated inflammation and insulin resistance affects actin cytoskeleton organization in podocytes. PLoS One. 2015;10:e0142291.

29. Yang W, Luo Y, Yang S, Zeng M, Zhang S, Liu J, et al. Ectopic lipid accumulation: potential role in tubular injury and inflammation in diabetic kidney disease. Clin Sci (Lond). 2018;132:2407-22.

30. Ding $Y$, Choi ME. Autophagy in diabetic nephropathy. J Endocrinol. 2015;224:R15-30.

31. Fang $L$, Zhou $Y, \mathrm{Cao}_{\mathrm{H}}$, Wen $\mathrm{P}$, Jiang $\mathrm{L}$, He W, et al. Autophagy attenuates diabetic glomerular damage through protection of hyperglycemia-induced podocyte injury. PLoS One. 2013;8:e60546.

32. Guo Z, Zhao Z. Effect of N-acetylcysteine on plasma adiponectin and renal adiponectin receptors in streptozotocin-induced diabetic rats. Eur J Pharm. 2007;558:208-13.

33. Park HS, Lim JH, Kim MY, Kim Y, Hong YA, Choi SR, et al. Resveratrol increases AdipoR1 and AdipoR2 expression in type 2 diabetic nephropathy. J Transl Med. 2016;14:176.

34. Okada-Iwabu M, Yamauchi T, Iwabu M, Honma T, Hamagami K, Matsuda K, et al. A small-molecule AdipoR agonist for type 2 diabetes and short life in obesity. Nature. 2013;503:493-9.

35. Li Y, Song B, Ruan C, Xue W, Zhao J. AdipoRon attenuates hypertension-induced epithelial-mesenchymal transition and renal fibrosis via promoting epithelial autophagy. J Cardiovasc Transl Res. 2021;14:538-45.

36. Wang Y, Liang B, Lau WB, Du Y, Guo R, Yan Z, et al. Restoring diabetes-induced autophagic flux arrest in ischemic/reperfused heart by ADIPOR (adiponectin receptor) activation involves both AMPK-dependent and AMPK-independent signaling. Autophagy. 2017;13:1855-69.

37. Yamauchi T, Nio Y, Maki T, Kobayashi M, Takazawa T, Iwabu M, et al. Targeted disruption of AdipoR1 and AdipoR2 causes abrogation of adiponectin binding and metabolic actions. Nat Med. 2007;13:332-9.

38. Iwabu M, Okada-Iwabu M, Yamauchi T, Kadowaki T. Adiponectin/adiponectin receptor in disease and aging. NPJ Aging Mech Dis. 2015;1:15013.

39. Lim JH, Kim HW, Kim MY, Kim TW, Kim EN, Kim Y, et al. Cinacalcet-mediated activation of the CaMKKbeta-LKB1-AMPK pathway attenuates diabetic nephropathy in $\mathrm{db} / \mathrm{db}$ mice by modulation of apoptosis and autophagy. Cell Death Dis. 2018;9:270.

40. Kitada M, Ogura Y, Monno I, Koya D. Regulating autophagy as a therapeutic target for diabetic nephropathy. Curr Diab Rep. 2017;17:53.

41. Yang D, Livingston MJ, Liu Z, Dong G, Zhang M, Chen JK, et al. Autophagy in diabetic kidney disease: regulation, pathological role and therapeutic potential. Cell Mol Life Sci. 2018;75:669-88.

42. Kim J, Kundu M, Viollet B, Guan KL. AMPK and mTOR regulate autophagy through direct phosphorylation of Ulk1. Nat Cell Biol. 2011;13:132-41.

43. Ro SH, Jung CH, Hahn WS, Xu X, Kim YM, Yun YS, et al. Distinct functions of Ulk1 and Ulk2 in the regulation of lipid metabolism in adipocytes. Autophagy. 2013;9:2103-14

44. Kaushik S, Rodriguez-Navarro JA, Arias E, Kiffin R, Sahu S, Schwartz GJ, et al. Autophagy in hypothalamic AgRP neurons regulates food intake and energy balance. Cell Metab. 2011;14:173-83.

45. Kim Y, Lim JH, Kim MY, Kim EN, Yoon HE, Shin SJ, et al. The Adiponectin receptor agonist adiporon ameliorates diabetic nephropathy in a model of type 2 diabetes. J Am Soc Nephrol. 2018;29:1108-27.

46. Tervaert TW, Mooyaart AL, Amann K, Cohen AH, Cook HT, Drachenberg CB, et al. Pathologic classification of diabetic nephropathy. J Am Soc Nephrol. 2010;21:556-63.
47. Sun L, Zhang D, Liu F, Xiang X, Ling G, Xiao L, et al. Low-dose paclitaxel ameliorates fibrosis in the remnant kidney model by down-regulating miR-192. J Pathol. 2011;225:364-77.

48. Ding $\mathrm{Y}$, Zhang $\mathrm{S}$, Yang $\mathrm{L}, \mathrm{Na} \mathrm{H}$, Zhang $\mathrm{P}$, Zhang $\mathrm{H}$, et al. Isolating lipid droplets from multiple species. Nat Protoc. 2013;8:43-51.

\section{ACKNOWLEDGEMENTS}

This work was supported by the Key Program of General Program of the National Natural Science Foundation of China (NSFC) (81730018), the National Key R\&D Program of China (2016YFC1305501; 2018YFC1314002)

\section{AUTHOR CONTRIBUTIONS}

$\mathrm{YH}, \mathrm{SX}, \mathrm{HZ}, \mathrm{SY}, \mathrm{MY}$, and NJ performed the experiments. $\mathrm{YH}$ and $\mathrm{SX}$ wrote the manuscript. $X Z, X X, P G, L W$, and $Y X$ provided the technical support and analyzed the data. $\mathrm{YH}, \mathrm{SX}, \mathrm{HZ}, \mathrm{SKY}, \mathrm{MY}, \mathrm{XZ}$, and NJ discussed the results of the study. LS designed, supervised, and revised the manuscript. LS is the guarantor of this work and had full access to all the study data and takes responsibility for its integrity and for the accuracy of the data analysis.

\section{COMPETING INTERESTS}

The authors declare no competing interests.

\section{ETHICS APPROVAL}

Ethical approval was obtained from the Ethics Committee of Second Xiangya Hospital, Central South University (Approval no. 2017-112).

\section{ADDITIONAL INFORMATION}

Correspondence and requests for materials should be addressed to Lin Sun.

Reprints and permission information is available at http://www.nature.com/ reprints

Publisher's note Springer Nature remains neutral with regard to jurisdictional claims in published maps and institutional affiliations.

\begin{abstract}
(i) Open Access This article is licensed under a Creative Commons Attribution 4.0 International License, which permits use, sharing, adaptation, distribution and reproduction in any medium or format, as long as you give appropriate credit to the original author(s) and the source, provide a link to the Creative Commons license, and indicate if changes were made. The images or other third party material in this article are included in the article's Creative Commons license, unless indicated otherwise in a credit line to the material. If material is not included in the article's Creative Commons license and your intended use is not permitted by statutory regulation or exceeds the permitted use, you will need to obtain permission directly from the copyright holder. To view a copy of this license, visit http://creativecommons. org/licenses/by/4.0/.
\end{abstract}

c The Author(s) 2021 